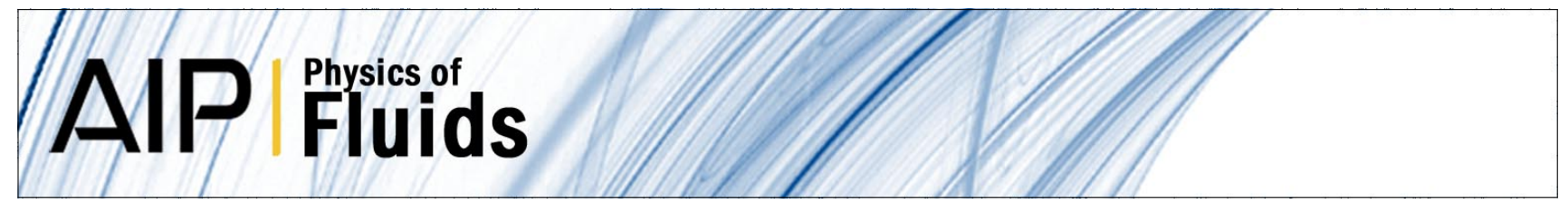

\title{
Physics-based analysis of the hydrodynamic stress in a fluid-particle system
}

Quan Zhang and Andrea Prosperetti

Citation: Phys. Fluids 22, 033306 (2010); doi: 10.1063/1.3365950

View online: http://dx.doi.org/10.1063/1.3365950

View Table of Contents: http://pof.aip.org/resource/1/PHFLE6/v22/i3

Published by the American Institute of Physics.

\section{Related Articles}

The dynamics of a vesicle in a wall-bound shear flow

Phys. Fluids 23, 121901 (2011)

A study of thermal counterflow using particle tracking velocimetry

Phys. Fluids 23, 107102 (2011)

Particle accumulation on periodic orbits by repeated free surface collisions

Phys. Fluids 23, 072106 (2011)

Drag force of a particle moving axisymmetrically in open or closed cavities

J. Chem. Phys. 135, 014904 (2011)

Modal and non-modal stability of particle-laden channel flow

Phys. Fluids 23, 064110 (2011)

\section{Additional information on Phys. Fluids}

Journal Homepage: http://pof.aip.org/

Journal Information: http://pof.aip.org/about/about_the_journal

Top downloads: http://pof.aip.org/features/most_downloaded

Information for Authors: http://pof.aip.org/authors

\section{ADVERTISEMENT}

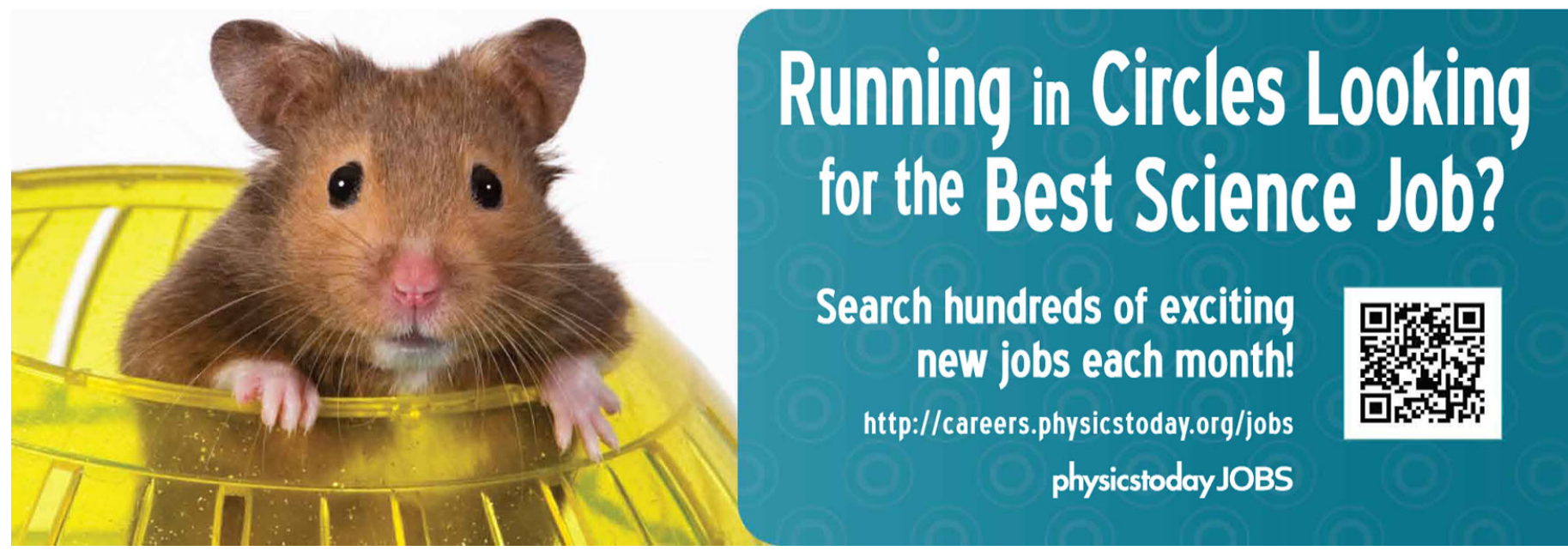




\title{
Physics-based analysis of the hydrodynamic stress in a fluid-particle system
}

\author{
Quan Zhang $^{\text {a) }}$ and Andrea Prosperetti ${ }^{\text {b) }}$ \\ Department of Mechanical Engineering, Johns Hopkins University, Baltimore, Maryland 21218, USA
}

(Received 12 February 2009; accepted 7 December 2009; published online 25 March 2010)

\begin{abstract}
The paper begins by showing how standard results on the average hydrodynamic stress in a uniform fluid-particle system follow from a direct, elementary application of Cauchy's stress principle. The same principle applied to the angular momentum balance proves the emergence, at the mesoscale, of an antisymmetric component of the volume-averaged hydrodynamic stress irrespective of the particle Reynolds number. Several arguments are presented to show the physical origin of this result and to explain how the averaging process causes its appearance at the mesoscale in spite of the symmetry of the microscale stress. Examples are given for zero and finite Reynolds number, and for potential flow. For this last case, the antisymmetric stress component vanishes, but the Cauchy principle proves nevertheless useful to derive in a straightforward way known results and to clarify their physical nature. (C) 2010 American Institute of Physics. [doi:10.1063/1.3365950]
\end{abstract}

\section{INTRODUCTION}

Due to their complexity, the theoretical description of most disperse multiphase flows of practical significancesediment transport, dust storms, fluidized beds, pneumatic conveying, slurries, suspensions, and many others-must rely necessarily on average-equations models. The closure of the equations obtained by formal averaging procedures has proven particularly intractable in the case of disperse flows due to a variety of factors such as nonlinearity of the equations, long-range particle-particle interactions, absence of a clear separation between micro- and macroscales, and others.

In envisaging a disperse fluid system as a complex continuum, one recognizes that the particle-fluid forces are internal to the mixture and therefore cancel in formulating a combined momentum equation for the two phases. The inhomogeneities affect convective momentum transport via Reynolds-like stresses and nonconvective transport via a mixture stress of hydrodynamic origin. We focus on the latter quantity and, in particular, on the particle contribution to it.

According to the stress principle of Cauchy, "upon any imagined closed surface $S$ there exists a distribution of stress vectors $\mathbf{t}$ whose resultant and moment are equivalent to those of the actual forces of material continuity exerted by the material outside $S$ upon that inside."1,2 Standard arguments (see, e.g., Refs. 2 and 3) then show that the stress vector is a linear function of the local normal, which leads to the introduction of the stress tensor.

In this paper we give an elementary and direct application of this principle, first, to the linear momentum balance for a disperse particle-fluid system treated as a continuum and show how it permits to recover the classic results for spatially uniform systems (Sec. III).

\footnotetext{
${ }^{a)}$ Present address: Dynaflow Inc., 10621-J Iron Bridge Road, Jessup, Maryland 20794. Electronic mail: zhq@jhu.edu.

${ }^{b)}$ Also at Faculty of Applied Science and Burgerscentrum, University of Twente, AE 7500 Enschede, The Netherlands. Electronic mail: prosperetti@jhu.edu.
}

A second application of the principle to the angular momentum balance leads to the identification of an antisymmetric contribution of hydrodynamic origin to the particle stress (Secs. IV and V). The nontrivial result here is that the volume-averaged hydrodynamic stress fails to be symmetric also when no external couples are exerted on the particles, provided spatial nonuniformities exist, e.g., of the particle volume fraction, the particle-mixture relative velocity or others.

This statement is to be interpreted in as follows. Let $\overline{\mathbf{s}}(\mathbf{x})$ denote the microscopic ensemble-averaged hydrodynamic stress at a geometric point $\mathbf{x}$ which is, of course, symmetric in the absence of body couples. Consider a mesoscopic volume $\Delta V$ large on the microscopic scale, but small on the macroscopic one. We wish to represent the integrated effect of $\overline{\mathbf{s}}$ in $\Delta V$ in terms of an effective stress $\boldsymbol{\Sigma}$ by writing

$$
\begin{aligned}
& \frac{1}{\Delta V} \int_{\Delta V} \boldsymbol{\nabla} \cdot \overline{\mathbf{s}} \mathrm{d}(\Delta V)=\boldsymbol{\nabla} \cdot \Sigma, \\
& \frac{1}{\Delta V} \int_{\Delta V} \mathbf{x} \times(\boldsymbol{\nabla} \cdot \overline{\mathbf{s}}) \mathrm{d}(\Delta V)=\mathbf{X} \times(\boldsymbol{\nabla} \cdot \Sigma),
\end{aligned}
$$

where $\mathbf{X}$ is the center of $\Delta V$. It is this effective stress tensor $\boldsymbol{\Sigma}$ which is not symmetric because, in a nonuniform system, the point of application of the resultant of the microscopic forces $\boldsymbol{\nabla} \cdot \overline{\mathbf{s}}$ does not coincide with the center of the volume. In other words, nonuniformities confer to the system qualities analogous to those of a structured continuum. As a consequence, the mesoscopic properties of the system are not reducible to simple ensemble averages at a point.

Although our method appears to be general, we focus specifically on the case of equal spherical particles. Our derivation applies equally well to Newtonian and non-Newtonian suspending fluids and arbitrary particle Reynolds numbers.

Some of these results have already been derived by ensemble-averaging techniques in earlier work. ${ }^{4,5}$ The main contribution of the present paper consists in its direct 
physical approach which gives a deeper insight into these matters addressing, among others, the question of how the averaging process results in a nonsymmetric stress in spite of the symmetry prevailing at the microscopic level (Secs. VI and $\mathrm{X}$; in particular, the relation with the earlier ensembleaverage derivation is discussed at the end of Sec. X). Furthermore, we provide several new examples: Stokes flow, potential flow, and dilute systems of particles at finite Reynolds numbers. The quantitative importance of the new effects that we discuss is demonstrated numerically by studying the sedimentation of a suspension "blob" in otherwise clear fluid (Sec. VIII). The specific closure that we use for this purpose is meant as an example only as our focus is the stress of hydrodynamic origin and not the formulation of a complete closed theory of balance laws and constitutive equations.

\section{LITERATURE REVIEW}

Previous studies of the average stress in a disperse fluidparticle system have not made a direct use of Cauchy's principle. Einstein's treatment of dilute viscous suspensions was based on a dissipation argument (see, e.g., Ref. 6). In his classic paper "The stress system in a suspension of force-free particles," Batchelor ${ }^{7}$ used ensemble averaging which he quickly converted to volume averaging by assuming spatial uniformity. He gave the particle contribution to the mixture stress in the form

$$
\frac{1}{V} \sum \int_{v} \boldsymbol{\sigma}^{P} \mathrm{~d} v
$$

where the integral is over the particle volume $v$ and the summation is extended to all the particles contained in the averaging volume $V$. Brenner ${ }^{8}$ used a combination of multiple scales and cell averaging to connect fluid mechanical principles to suspension mechanics. None of these approaches directly addresses the concept of stress as force transmitted across a surface. This point is only alluded to on p. 552 of Batchelor's paper ${ }^{7}$ without elaboration.

Almog and Brenner ${ }^{9}$ mention Cauchy's principle in their title for the special case in which the particle rotation is caused by a nonuniform weight distribution in its interior. However, the reference is to Cauchy's balance of angular momentum [see Eq. (24) below], rather than to the force transmitted through surface elements as here.

A different approach to the calculation of the stress tensor in a viscous suspension was taken in several papers by Felderhof and co-workers (see, e.g., Refs. 10 and 11) and Bedeaux, Beenakker and co-workers (e.g., Refs. 12 and 13; see also the review in Refs. 14 and 15). In their approach the average stress is identified with the argument of a divergence operator appearing in the mixture momentum equation. The divergence theorem connects this quantity to a surface traction, but leaves open the question of possible divergence-free contributions to the stress, as noted below at the end of Sec. V.

The vast majority of past work has dealt with statistically uniform systems of force- and couple-free particles. As we show below, in these conditions the stress is symmetric unless the particles are subject to an external couple as found by many authors (see, e.g., Refs. 7 and 8). Leal, ${ }^{16}$ and especially Brenner and co-workers, have devoted a considerable attention to this couple-induced antisymmetry. ${ }^{8,17-19}$

The literature on the stress tensor in the presence of significant inertial effects is much less plentiful. To some extent, the close connection between fundamental theory and mathematical model that has been achieved in the Stokes flow case is found in the potential flow regime (see, e.g., Refs. 20-27). In this context the words "particle stress" have been used in a sense which is not quite in keeping with the Cauchy point of view. This issue is addressed in Sec. IX.

\section{STRESS IN A UNIFORM SYSTEM}

In terms of the exact fields in the two phases, the overall momentum balance for a macroscopic control volume $\mathcal{V}$ bounded by a surface $\mathcal{S}$ is

$$
\begin{aligned}
\int_{\mathcal{V}}\left[(1-\chi) \rho^{F} \mathbf{a}^{F}+\chi \rho^{P} \mathbf{a}^{P}\right] \mathrm{d} \mathcal{V} \\
=\oint_{\mathcal{S}}\left[(1-\chi) \boldsymbol{\sigma}^{F}+\chi \boldsymbol{\sigma}^{P}\right] \cdot \mathbf{N} \mathrm{d} \mathcal{S} \\
\quad+\int_{\mathcal{V}}\left[(1-\chi) \rho^{F}+\chi \rho^{P}\right] \mathbf{g} \mathrm{d} \mathcal{V} .
\end{aligned}
$$

Here $\rho$ is the density, a the acceleration, $\mathbf{g}$ the body force per unit mass (assumed equal for the two phases) and $\boldsymbol{\sigma}$ the stress tensor. Superscripts $F$ and $P$ refer to the fluid and particle phases, respectively, $\chi$ is the characteristic, or indicator, function of the particle phase and $\mathbf{N}$ is the unit outward normal. We wish to express the stress transmitted across the surface $\mathcal{S}$ in terms of an average stress $\boldsymbol{\Sigma}$ defined by

$$
\boldsymbol{\Sigma}=\left\langle(1-\chi) \boldsymbol{\sigma}^{F}\right\rangle+\left\langle\chi \boldsymbol{\sigma}^{P}\right\rangle=(1-\beta)\left\langle\boldsymbol{\sigma}^{F}\right\rangle+\beta\left\langle\boldsymbol{\sigma}^{P}\right\rangle,
$$

in which $\beta=\langle\chi\rangle$ is the volume fraction (equal to the area fraction, see, e.g., Refs. 28 and 29) of the disperse phase and $\left\langle\boldsymbol{\sigma}^{F}\right\rangle,\left\langle\boldsymbol{\sigma}^{P}\right\rangle$ are the average contributions of each phase over a mesoscale surface element $\Delta \mathcal{S}$.

To calculate the average particle contribution $\beta\left\langle\boldsymbol{\sigma}^{P}\right\rangle$ from Cauchy's principle, we consider the average force transmitted through the particles cut across by the surface element $\Delta \mathcal{S}$, which we take planar for simplicity. As pointed out by Batchelor, ${ }^{7}$ this surface element must be such that it "makes an unbiased sample of the suspension and [has] linear dimensions large compared with the average particle spacing. In the particular case of the stress, an average over a plane surface (which cuts through both ambient fluid and particles)... has obvious appeal as a way of defining average stress" (see also Ref. 9). Invoking a surface element with these properties is a standard procedure in spatial averaging and has been used, among others, by O'Brien ${ }^{28}$ (see also, e.g., Refs. 30 and 31).

The centers of the particles contributing to $\left(\left\langle\boldsymbol{\sigma}^{P}\right\rangle \cdot \mathbf{N}\right) \Delta \mathcal{S}$ are contained in a cylinder $\mathcal{C}$ based on $\Delta \mathcal{S}$ and protruding by amounts equal to the particle radius $a$ on each side of it (Fig. 1) so that 


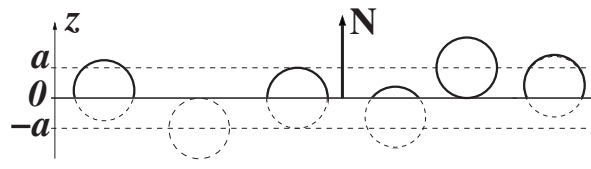

FIG. 1. Averaging volume for the calculation of the particle stress by Cauchy's stress principle.

$$
\beta \Delta \mathcal{S}\left\langle\boldsymbol{\sigma}^{P}\right\rangle \cdot \mathbf{N}=\left\langle\sum_{j \in \mathcal{C}} \int_{S_{\text {cut }}^{j}} \boldsymbol{\sigma}^{P} \cdot \mathbf{N} \mathrm{d} S_{\text {cut }}^{j}\right\rangle .
$$

The summation is extended to all the particles which intersect $\Delta \mathcal{S}$ and the integrals are over the portion of $\Delta \mathcal{S}$ cutting across the $j$ th particle denoted by $S_{\text {cut }}^{j}$.

If the use of spatial averaging is justified and the hypothesis of separation of scales is satisfied, the number of particles with center in a "slice" of the cylinder of volume $\Delta \mathcal{S} \mathrm{d} z$ can be taken to be $n \Delta \mathcal{S} \mathrm{d} z$, where $n$ is the particle number density. In these conditions we can replace the summation in Eq. (6) by an integral over the volume of the cylinder $\mathcal{C}$ :

$$
\begin{aligned}
& \left\langle\sum_{j \in \mathcal{C}} \int_{S_{\text {cut }}^{j}} \boldsymbol{\sigma}^{P} \cdot \mathbf{N} \mathrm{d} S_{\text {cut }}^{j}\right\rangle \\
& \quad=\Delta \mathcal{S}\left\langle\int_{-a}^{a} \mathrm{~d} z n \int_{S_{\text {cut }}} \boldsymbol{\sigma}^{P} \cdot \mathbf{N} \mathrm{d} S_{\text {cut }}\right\rangle .
\end{aligned}
$$

Here the coordinate $z$ is parallel to $\mathbf{N}$ with $z=0$ on $\Delta \mathcal{S}$ (Fig. 1). For a locally uniform system $n$ is a constant and we therefore find

$$
\beta \Delta \mathcal{S}\left\langle\boldsymbol{\sigma}^{P}\right\rangle=n \Delta \mathcal{S}\left\langle\int_{-a}^{a} \mathrm{~d} z \int_{S_{\text {cut }}} \boldsymbol{\sigma}^{P} \mathrm{~d} S_{\text {cut }}\right\rangle .
$$

This expression has been derived by keeping $\Delta \mathcal{S}$ fixed and averaging over all the particles that straddle it. But, because of uniformity, the stress distribution inside each particle is statistically the same. Thus, instead of considering the various contributions $S_{\text {cut }} \mathrm{d} z$, each from a different particle, we may equivalently sum the contributions from a single particle (for an alternative interpretation, see Fig. 2). With this remark, then, we may write

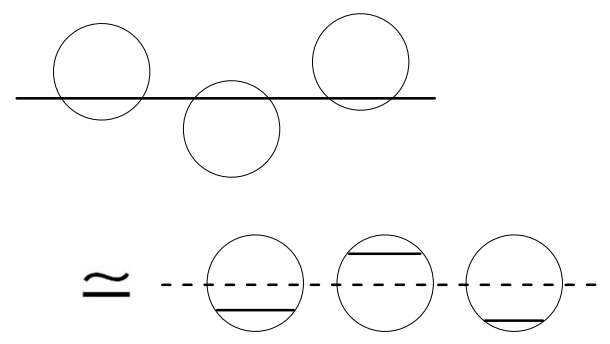

FIG. 2. Heuristic argument for an alternative derivation of Eq. (9). Instead of keeping $\Delta \mathcal{S}$ fixed and averaging over all the particles that straddle it, we can consider a fixed particle and all the possible $\Delta \mathcal{S}$ 's cutting through it. Since $\boldsymbol{\sigma}^{P}$ is only a function of the distance of the particle center from $S_{\text {cut }}$, it is immaterial whether one thinks of executing the integration by moving $S_{\text {cut }}$ or the particle center.

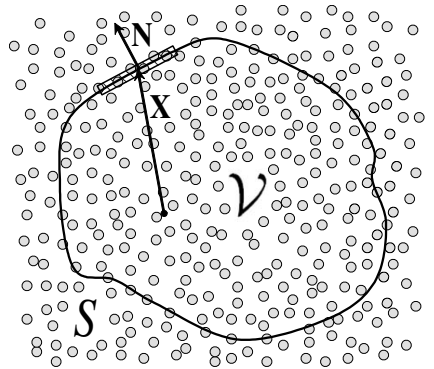

(a)

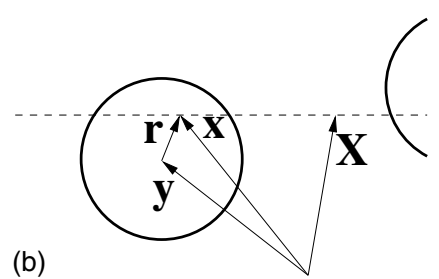

FIG. 3. (a) A control volume in the fluid-particle mixture. (b) Geometry for the derivation of Eq. (13).

$$
\left\langle\int_{-a}^{a} \mathrm{~d} z \int_{S_{\text {cut }}} \boldsymbol{\sigma}^{P} \mathrm{~d} S_{\text {cut }}\right\rangle=\left\langle\int_{v} \boldsymbol{\sigma}^{P} \mathrm{~d} v\right\rangle,
$$

where the integral is now over the volume $v$ of a particle and the angle brackets average over all the particles in the cylin$\operatorname{der} \mathcal{C}$. We have thus found the result

$$
\beta\left\langle\boldsymbol{\sigma}^{P}\right\rangle=n\left\langle\int_{v} \boldsymbol{\sigma}^{P} \mathrm{~d} v\right\rangle,
$$

which is essentially Eq. (3) and Eq. (4.1) in Batchelor. ${ }^{7}$ In a volume-averaging context this relation is rather obvious and could be written down intuitively with no need for a proof. Nevertheless, our simple argument shows that it is consistent with Cauchy's principle (which, as we have seen, essentially involves a surface averaging procedure) and it will be useful in the less obvious case of the couple transmitted across $\Delta \mathcal{S}$ taken up in the next section.

\section{ANGULAR MOMENTUM}

The force transmitted by the particles across the boundary $\mathcal{S}$ of a macroscopic control volume $\mathcal{V}$ contributes not only to the linear momentum of the mixture inside the control volume, but also to its angular momentum according to

$$
\oint_{\mathcal{S}}\left[(1-\beta)\left\langle\mathbf{x} \times \boldsymbol{\sigma}^{F}\right\rangle+\beta\left\langle\mathbf{x} \times \boldsymbol{\sigma}^{P}\right\rangle\right] \cdot \mathbf{N d} \mathcal{S},
$$

where $\mathbf{x}$ is the exact position vector of each microscopic surface element, not necessarily equal to $\mathbf{X}$, which is the position vector of the surface element $\Delta \mathcal{S}$ (Fig. 3). Note that $\mathbf{x}$ depends on how the particle is intersected by $\mathcal{S}$ and is, therefore, a statistical quantity so that $\left\langle\mathbf{x} \times \boldsymbol{\sigma}^{P}\right\rangle \neq \mathbf{X} \times\left\langle\boldsymbol{\sigma}^{P}\right\rangle$. Similarly to Eq. (7), the second term gives contributions 


$$
\begin{aligned}
\beta \Delta \mathcal{S}\left\langle\mathbf{x} \times \boldsymbol{\sigma}^{P}\right\rangle \cdot \mathbf{N} \\
\quad=\Delta \mathcal{S}\left\langle\int_{-a}^{a} n \mathrm{~d} z \int_{S_{\text {cut }}} \mathbf{x} \times\left(\boldsymbol{\sigma}^{P} \cdot \mathbf{N}\right) \mathrm{d} S_{\text {cut }}\right\rangle .
\end{aligned}
$$

We write $\mathbf{x}=\mathbf{r}+\mathbf{y}$, where $\mathbf{r}=\mathbf{x}-\mathbf{y}$ is the position vector of the integration point on $S_{\text {cut }}$ with respect to the particle center at y [Fig. 3(b)]. Substituting into Eq. (12), we find

$$
\begin{aligned}
\beta \Delta \mathcal{S}\left\langle\mathbf{x} \times \boldsymbol{\sigma}^{P}\right\rangle \cdot \mathbf{N} \\
=\Delta \mathcal{S}\left\langle\int_{-a}^{a} n \mathrm{~d} z \int_{S_{\mathrm{cut}}} \mathbf{r} \times\left(\boldsymbol{\sigma}^{P} \cdot \mathbf{N}\right) \mathrm{d} S_{\mathrm{cut}}\right\rangle \\
+\Delta \mathcal{S} \mathbf{X} \times\left\langle\int_{-a}^{a} n \mathrm{~d} z \int_{S_{\mathrm{cut}}}\left(\boldsymbol{\sigma}^{P} \cdot \mathbf{N}\right) \mathrm{d} S_{\mathrm{cut}}\right\rangle \\
=\Delta \mathcal{S}\left\langle\int_{-a}^{a} n \mathrm{~d} z \int_{S_{\mathrm{cut}}} \mathbf{r} \times\left(\boldsymbol{\sigma}^{P} \cdot \mathbf{N}\right) \mathrm{d} S_{\mathrm{cut}}\right\rangle \\
+\Delta \mathcal{S} \beta \mathbf{X} \times\left\langle\boldsymbol{\sigma}^{P} \cdot \mathbf{N}\right\rangle .
\end{aligned}
$$

In the first step, we have replaced the average of $\mathbf{y} \times\left(\boldsymbol{\sigma}^{P} \cdot \mathbf{N}\right)$ by $\mathbf{X} \times\left\langle\boldsymbol{\sigma}^{P}\right\rangle \cdot \mathbf{N}$, which is legitimate, as there is a statistically equal number of particles with centers above and below $\Delta \mathcal{S}$ and to the left and to the right of the center $\mathbf{X}$ of $\Delta \mathcal{S}$. By Eq. (8), the resulting integral is just the average surface traction. The first term in Eq. (13) can now be evaluated as before with the result

$$
\begin{gathered}
\left\langle\int_{-a}^{a} n \mathrm{~d} z \int_{S_{\mathrm{cut}}} \mathbf{r} \times\left(\boldsymbol{\sigma}^{P} \cdot \mathbf{N}\right) \mathrm{d} S_{\mathrm{cut}}\right\rangle \\
=n\left\langle\int_{v} \mathbf{r} \times\left(\boldsymbol{\sigma}^{P} \cdot \mathbf{N}\right) \mathrm{d} v\right\rangle .
\end{gathered}
$$

The arguments that follow show that this term is equivalent to a nonconvective couple flux due to the action of the particle material outside the control volume on that inside. The physical origin of the effect is discussed in Secs. VI and X.

To develop this term further, we follow a standard procedure $^{6,7}$ using the divergence theorem, the continuity of the stress at the particle surface and the identity

$$
\epsilon_{i j k} r_{j} \sigma_{k l}^{P}=\epsilon_{i j k}\left[\frac{\partial}{\partial r_{m}}\left(r_{j} r_{l} \sigma_{k m}^{P}\right)-r_{j} r_{l} \frac{\partial \sigma_{k m}^{P}}{\partial r_{m}}\right],
$$

which relies on the symmetry of $\boldsymbol{\sigma}^{P}$ (Ref. 32) to write

$$
\begin{aligned}
\int_{v} \mathbf{r} & \times\left(\boldsymbol{\sigma}^{P} \cdot \mathbf{N}\right) \mathrm{d} v \\
= & \oint(\mathbf{N} \cdot \mathbf{r}) \mathbf{r} \times\left(\boldsymbol{\sigma}^{F} \cdot \mathbf{n}\right) \mathrm{d} S \\
& -\int_{v}(\mathbf{N} \cdot \mathbf{r}) \mathbf{r} \times\left(\boldsymbol{\nabla} \cdot \boldsymbol{\sigma}^{P}\right) \mathrm{d} v,
\end{aligned}
$$

where the first integral in the right-hand side is over the particle surface, with unit outward normal n. The last term can be further manipulated by using the momentum equation for the particle material with the result

$$
\int_{v}(\mathbf{N} \cdot \mathbf{r}) \mathbf{r} \times\left(\boldsymbol{\nabla} \cdot \boldsymbol{\sigma}^{P}\right) \mathrm{d} v=\int_{v} \rho^{P}(\mathbf{N} \cdot \mathbf{r}) \mathbf{r} \times\left(\mathbf{a}^{P}-\mathbf{g}\right) \mathrm{d} v,
$$

in which, as before, $\rho^{P}$ and $\mathbf{a}^{P}$ are the local particle-material density and acceleration and $\mathbf{g}$ is the body force. The case of an essentially rigid, homogeneous particle is of particular interest as then we have

$$
\rho^{P} \int_{v}(\mathbf{N} \cdot \mathbf{r}) \mathbf{r} \times\left(\mathbf{a}^{P}-\mathbf{g}\right) \mathrm{d} v=\frac{1}{5} a^{2} m^{P} \mathbf{N} \times(\dot{\mathbf{w}}-\mathbf{g}),
$$

in which $m^{P}=\rho^{P} v$ is the particle mass and $\dot{\mathbf{w}}$ the acceleration of its center of mass. From the particle equation of motion this expression must be proportional to the hydrodynamic force on the particle so that

$$
\int_{v}(\mathbf{N} \cdot \mathbf{r}) \mathbf{r} \times\left(\boldsymbol{\nabla} \cdot \boldsymbol{\sigma}^{P}\right) \mathrm{d} v=\frac{1}{5} a^{2} \mathbf{N} \times \oint \boldsymbol{\sigma}^{F} \cdot \mathbf{n} \mathrm{d} S .
$$

The average of Eq. (16) may then be written as

$$
n\left\langle\int_{v} \mathbf{r} \times\left(\boldsymbol{\sigma}^{P} \cdot \mathbf{N}\right) \mathrm{d} v\right\rangle=\mathrm{C} \cdot \mathbf{N},
$$

where the couple flux tensor $\mathrm{C}$ is given by

$$
\mathrm{C}_{i j}=n \epsilon_{i k l}\left\langle\oint r_{j} r_{k}\left(\boldsymbol{\sigma}^{F} \cdot \mathbf{n}\right)_{l} \mathrm{~d} S-\frac{1}{5} a^{2} \delta_{j k} \oint\left(\boldsymbol{\sigma}^{F} \cdot \mathbf{n}\right)_{l} \mathrm{~d} S\right\rangle .
$$

This agrees with the result found by a different method in Ref. 5. In the above derivation we have not included the effect of interparticle forces. This aspect is taken up in Appendix A. In particular, when these forces are uniformly distributed in the particle, their contribution to the couple flux tensor is found to vanish.

We thus conclude that the angular momentum imparted by the surface stresses to the mixture material inside the control volume is, from Eqs. (11), (13), and (20),

$$
\begin{aligned}
\oint_{\mathcal{S}} & {\left[(1-\beta)\left\langle\mathbf{x} \times \boldsymbol{\sigma}^{F}\right\rangle+\beta\left\langle\mathbf{x} \times \boldsymbol{\sigma}^{P}\right\rangle\right] \cdot \mathbf{N d} S } \\
= & \oint_{\mathcal{S}}\left[(1-\beta) \mathbf{X} \times\left\langle\boldsymbol{\sigma}^{F}\right\rangle+\beta \mathbf{X} \times\left\langle\boldsymbol{\sigma}^{P}\right\rangle+\mathrm{C}\right] \cdot \mathbf{N} \mathrm{d} S \\
= & \int_{\mathcal{V}}\left\{\mathbf{X} \times \boldsymbol{\nabla} \cdot\left[(1-\beta)\left\langle\boldsymbol{\sigma}^{F}\right\rangle+\beta\left\langle\boldsymbol{\sigma}^{P}\right\rangle\right]\right. \\
& \left.-\beta \boldsymbol{\epsilon} \cdot\left\langle\boldsymbol{\sigma}^{P}\right\rangle+\boldsymbol{\nabla} \cdot \mathrm{C}\right\} \mathrm{d} \mathcal{V},
\end{aligned}
$$

in writing which we have used the fact that the average fluid stress $\left\langle\boldsymbol{\sigma}^{F}\right\rangle$ is symmetric. ${ }^{33}$ This expression must equal the rate of change of the angular momentum of the mixture material in $\mathcal{V}$, minus a total external applied couple which might act on the particles. If the average velocities are defined by averaging the microscopic velocities $\mathbf{u}^{F}$ and $\mathbf{u}^{P}$, the mixture has no intrinsic angular momentum. In this case, the first term in the right-hand side of Eq. (22) exactly balances the rate of change of the angular momentum and the couple due 
to the body force (Appendix B). The remaining terms, namely, the antisymmetric part of the stress, the divergence of the couple flux, and the external body couple $\mathbf{L}$ must balance each other and we recover the well-known balance equation (see, e.g., Ref. 3, pp. 103-104)

$$
\beta \epsilon_{i j k}\left\langle\boldsymbol{\sigma}^{P}\right\rangle_{j k}=\frac{\partial \mathrm{C}_{i j}}{\partial x_{j}}+n L_{i}
$$

For a nonpolar fluid, the fluid stress gives no contribution to the antisymmetric part of the mesoscopic average stress, and we may equally well write this equation in the form

$$
\epsilon_{i j k} \Sigma_{j k}=\frac{\partial \mathrm{C}_{i j}}{\partial x_{j}}+n L_{i} .
$$

[The easiest way to see that the fluid stress gives no contribution to the antisymmetric part of the mesoscopic average stress is to note that the fluid may be thought of as constituted of "particles" with a vanishingly small size. The same argument used for the real particles would then lead to a result such as Eq. (21) with the integral extended to a vanishingly small volume.]

In an ordinary unstructured continuum $C$ vanishes and $\mathbf{L}=0$ in the absence of external body couples. This equation embodies then the familiar argument used to prove the symmetry of the stress tensor (see, e.g., Ref. 3). Our result shows that in a fluid-particle system, even in the absence of external couples, the antisymmetric part of the mesoscopic stress only vanishes if $\boldsymbol{\nabla} \cdot \mathrm{C}=0$, e.g., in a uniform system (in which, however, $C$ may well be nonzero). Since, according to Eq. (24), the antisymmetric component is exactly balanced by the divergence of the couple flux, it is not a source of angular momentum for the mixture, although it is a source of linear momentum through its contribution to $\boldsymbol{\nabla} \cdot \boldsymbol{\Sigma}$.

In a micropolar fluid the couple flux $C$ arises from the presence of couple stresses (see, e.g., Ref. 3). In our case no such stresses are present at the microscopic level. Here, as explained in qualitative terms in Sec. $\mathrm{X}$, the couple flux is a consequence of nonuniformities in the hydrodynamic stresses exerted by the fluid on the particles.

From Eq. (23) we have the antisymmetric part of $\left\langle\boldsymbol{\sigma}^{P}\right\rangle$

$$
\begin{aligned}
\beta\left\langle\boldsymbol{\sigma}^{P}\right\rangle_{p q}^{A} & \equiv \frac{1}{2} \beta\left(\left\langle\boldsymbol{\sigma}^{P}\right\rangle_{p q}-\left\langle\boldsymbol{\sigma}^{P}\right\rangle_{q p}\right) \\
& =\frac{1}{2} \frac{\partial}{\partial x_{j}} \epsilon_{i p q} \mathrm{C}_{i j}+\frac{1}{2} n \epsilon_{i p q} L_{i},
\end{aligned}
$$

which (up to smaller terms, see Appendix C) can be written as

$$
\beta\left\langle\boldsymbol{\sigma}^{P}\right\rangle_{p q}^{A}=\epsilon_{p q i}\left(\frac{1}{2} n L_{i}\right)-\epsilon_{p q i}(\boldsymbol{\nabla} \times \mathbf{V})_{i}+\frac{1}{2} \partial_{k}\left(\Delta_{p k q}-\Delta_{q k p}\right),
$$

where

$$
\begin{aligned}
\mathbf{V}=- & \frac{1}{10} n a^{2}\left\langle\oint(\mathbf{I}-\mathbf{n n}) \cdot\left(\boldsymbol{\sigma}^{F} \cdot \mathbf{n}\right) \mathrm{d} S\right\rangle, \\
\Delta_{p k q}= & \frac{1}{3}\left(T_{q p k}-T_{k p q}\right) \\
& +\frac{1}{15}\left[\delta_{p q}\left(T_{k}-4 T_{j j k}\right)-\delta_{p k}\left(T_{q}-4 T_{j j q}\right)\right],
\end{aligned}
$$

with

$$
\begin{aligned}
& T_{k}=n a^{2}\left\langle\oint\left(\boldsymbol{\sigma}^{F} \cdot \mathbf{n}\right)_{k} \mathrm{~d} S\right\rangle, \\
& T_{k p q}=n a^{2}\left\langle\oint\left(\boldsymbol{\sigma}^{F} \cdot \mathbf{n}\right)_{k} n_{p} n_{q} \mathrm{~d} S\right\rangle .
\end{aligned}
$$

In this and the previous section, we have assumed local uniformity in the neighborhood of the surface element. A different derivation for a slightly inhomogeneous system is given in Appendix C.

\section{THE ANTISYMMETRIC STRESS}

As given by Eq. (26), the complete antisymmetric stress consists of three contributions, each one with a different physical origin. The first contribution, already known from the work of Batchelor ${ }^{7}$ and Brenner, ${ }^{8}$ arises from the external couple applied to the particles and will not be discussed further (see also Ref. 5).

The second contribution is the curl of the vector $\mathbf{V}$ defined in Eq. (27), which may be rewritten as

$$
\begin{aligned}
\mathbf{V} & =-\frac{1}{10} n a^{2}\langle\oint(\mathbf{I}-\mathbf{n n}) \cdot(\boldsymbol{\tau} \cdot \mathbf{n}) \mathrm{d} S\rangle \\
& =\frac{1}{10} n a^{2}\langle\oint \mathbf{n} \times[\mathbf{n} \times(\boldsymbol{\tau} \cdot \mathbf{n})] \mathrm{d} S\rangle,
\end{aligned}
$$

where $\boldsymbol{\tau}=\boldsymbol{\sigma}^{F}-(1 / 3)\left(\operatorname{Tr} \boldsymbol{\sigma}^{F}\right) \mathbf{I}$ is the deviatoric part of the fluid stress. In an incompressible Newtonian fluid, this would be the viscous stress, and it would therefore vanish in a perfect fluid. If the order of magnitude of $|\boldsymbol{\tau} \cdot \mathbf{n}|$ is estimated as $\mu U_{\text {rel }} / \delta$, in which $U_{\text {rel }}$ is a measure of the relative particlefluid velocity and $\delta$ a boundary layer thickness, we find, in order of magnitude,

$$
V \sim \mu \beta \frac{a}{\delta} U_{\mathrm{rel}}
$$

For Stokes flow $\delta \sim a$ while, at sufficiently large Reynolds number, $a / \delta \sim \sqrt{\operatorname{Re}}$, with $\operatorname{Re}$ the Reynolds number of the relative motion. In this latter case

$$
V \sim \mu \beta \sqrt{\operatorname{Re}} U_{\text {rel }}
$$

By making specific assumptions on the nature of the flow and the particle distribution we can derive more specific results. 
TABLE I. Numerically computed $\mu_{V} / \mu$ to first order in the particle volume fraction for different particle Reynolds numbers.

\begin{tabular}{lcc}
\hline \hline & $\mu_{V} /(\beta \mu)$ & $\mu_{V} /(\beta \mu \sqrt{\operatorname{Re}})$ \\
\hline $\operatorname{Re}=0$ & 0.3 & $\ldots$ \\
$\operatorname{Re}=5$ & 0.457 & 0.204 \\
$\operatorname{Re}=10$ & 0.535 & 0.169 \\
$\operatorname{Re}=20$ & 0.649 & 0.145 \\
$\operatorname{Re}=25$ & 0.694 & 0.139 \\
$\operatorname{Re}=30$ & 0.734 & 0.134 \\
$\operatorname{Re}=40$ & 0.803 & 0.127 \\
$\operatorname{Re}=50$ & 0.861 & 0.122 \\
\hline \hline
\end{tabular}

As a first example, we consider the dilute limit at finite Reynolds number with negligible ambient shear. In this limit, $\mathbf{V}$ can be calculated by considering an isolated particle and the result has the form

$$
\mathbf{V}=\mu_{V}\left(\overline{\mathbf{w}}-\left\langle\mathbf{u}^{F}\right\rangle\right)
$$

where $\mu_{V}$ is a new viscosity parameter and $\overline{\mathbf{w}}$ is the average particle translational velocity. (Here and in the following, the overline denotes the average calculated over all the particles the center of which is inside the averaging volume.) The viscosity parameter $\mu_{V}$ is proportional to $\beta$ and, from Oseen's solution we find, correct to first order in Re,

$$
\frac{\mu_{V}}{\mu}=\frac{3}{10} \beta\left[1+\frac{3}{8} \operatorname{Re}\right],
$$

with $\operatorname{Re}=2 a \rho^{F}\left|\overline{\mathbf{w}}-\left\langle\mathbf{u}^{F}\right\rangle\right| / \mu$. At larger values of $\mathrm{Re}$, a numerical calculation carried out using the PHYSALIS numerical method $^{34}$ gives the results shown in Table I and in Fig. 4. The line is a fit $\mu_{V} /(\beta \mu)=3 / 10+0.08 \sqrt{\mathrm{Re}}$. Although this relatively crude fit does not capture the Oseen term, the anticipated scaling (32) is approximately verified.

At finite particle concentration the relevant ambient velocity is the mixture volumetric flux $\mathbf{u}_{m}$ defined by

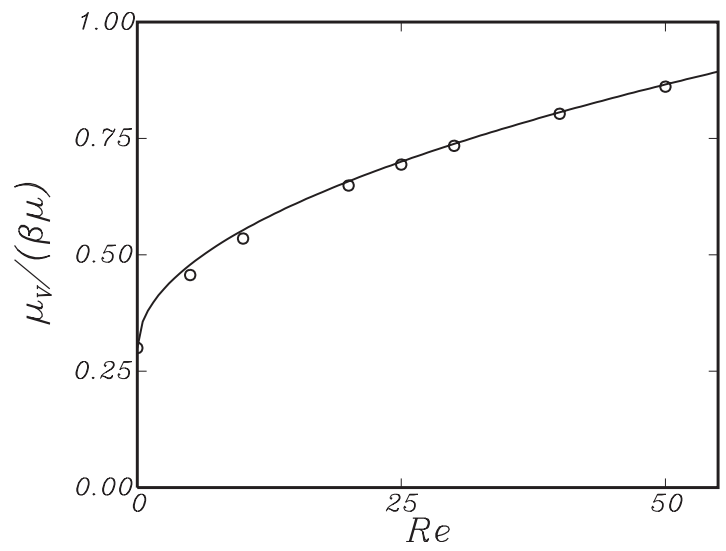

FIG. 4. The polar viscosity parameter $\mu_{V}$ normalized by $\beta \mu$ as a function of the particle Reynolds number for a dilute system. The line is the fit $\mu_{V} /(\beta \mu)=3 / 10+0.08 \sqrt{\mathrm{Re}}$. The circles are the numerical values given in Table I.
TABLE II. Numerically computed $\zeta$ defined in Eq. (38) and $\mu_{V} / \mu$ vs particle volume fraction $\beta$ for Stokes flow.

\begin{tabular}{ccc}
\hline \hline$\beta$ & $\zeta$ & \\
$(\%)$ & $\zeta$ & $\mu_{V} / \mu$ \\
\hline 1 & 3.56 & 0.0032 \\
2 & 3.55 & 0.0067 \\
3 & 3.53 & 0.0106 \\
5 & 3.55 & 0.0196 \\
10 & 3.54 & 0.0509 \\
15 & 3.53 & 0.0990 \\
20 & 3.53 & 0.1705 \\
25 & 3.50 & 0.2750 \\
30 & 3.46 & 0.4258 \\
35 & 3.94 & 0.5762 \\
40 & 3.37 & 0.9480 \\
45 & 3.36 & 1.3684 \\
\hline \hline
\end{tabular}

$$
\mathbf{u}_{m}=(1-\beta)\left\langle\mathbf{u}^{F}\right\rangle+\beta^{P}\left\langle\mathbf{u}^{P}\right\rangle,
$$

so that (assuming isotropy)

$$
\mathbf{V}=\mu_{V}\left(\overline{\mathbf{w}}-\mathbf{u}_{m}\right) \text {. }
$$

A numerical investigation of the dependence of the parameter $\mu_{V}$ on the particle volume fraction $\beta$ carried out on the assumption that the particles are distributed according to the hard-sphere distribution function (which does not include flow-dependent features), gives, for $\operatorname{Re}=0,{ }^{5}$

$$
\frac{\mu_{V}}{\mu}=\frac{\beta[3-\beta \zeta(\beta)]}{10 H(\beta)} .
$$

Here $H(\beta)$ is the hindrance function for sedimentation and $\zeta \simeq 3.5$ has a weak dependence on $\beta$; the two quantities are well represented by the fits

$$
\zeta \simeq 3.54\left(1-0.214 \beta^{2}\right), \quad H \simeq(1-\beta)^{6.55-3.34 \beta} .
$$

Numerical values for $\zeta$ and $\mu_{V} / \mu$ are provided in Table II. The determining effect of a force $\mathbf{F}$ applied to the particles in generating a nonzero $\mathbf{V}$ is evident from the fact that, by definition of the hindrance function, $\left(\overline{\mathbf{w}}-\mathbf{u}_{m}\right) / H$ is proportional to it. An interesting point to be made concerning the expression (37) for $\mu_{V} / \mu$ is that, provided $\beta$ is kept constant, this quantity remains finite in the continuum limit in which the particle size becomes infinitesimally small compared with the macroscopic length.

The third contribution to the antisymmetric stress in Eq. (26), (1/2) $\partial_{k}\left(\Delta_{p k q}-\Delta_{q k p}\right)$, when combined with a corresponding one from the symmetric stress, gives $\partial_{k} \Delta_{p k q}$ which, as is clear from the definition (28) of $\Delta_{p k q}$, has a zero double divergence and, therefore, does not contribute to the linear momentum of the mixture (see Ref. 5). For this reason this term was neglected in our earlier study. However, its omission would be incorrect if one were to consider, for example, a condition of continuity of the stress across an interface separating two disperse flows. (This is an example of the possible shortcomings associated with the identification of the stress with the argument of the divergence operator in the 


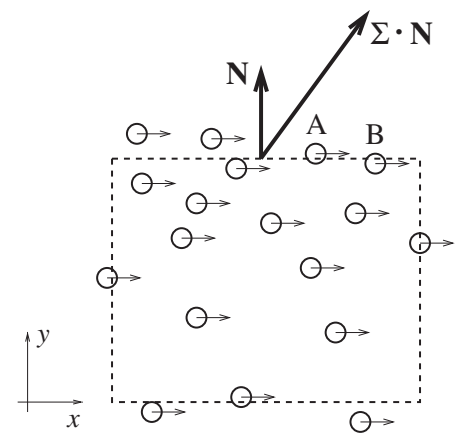

(a)

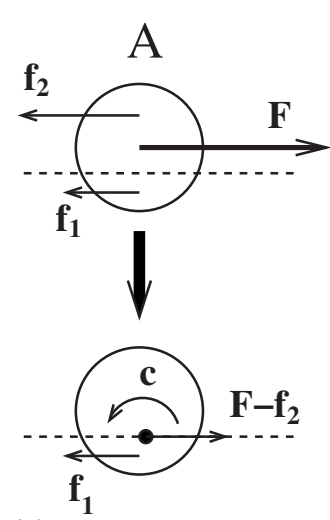

(b)

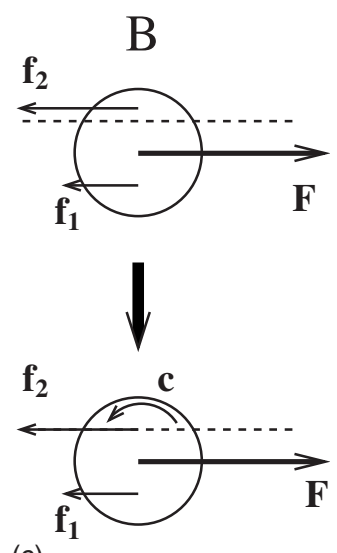

(c)

FIG. 5. Qualitative explanation of the physical origin of the antisymmetric stress. Consider particles such as $A$ and $B$ subjected to an external force $\mathbf{F}$ and straddling the surface of a volume element, and neglect inertia for simplicity. The force $\mathbf{F}$ is resisted by hydrodynamic forces $\mathbf{f}_{1}$ and $\mathbf{f}_{2}$ arising from the part of the particle surface inside and outside the control volume. For a particle such as $A$ (lower left), the dynamical effect of the external portion of the particle on the internal one can be represented by a resultant force $\mathbf{F}-\mathbf{f}_{2}^{A}$ and a couple $\mathbf{c}^{A}$. For a particle such as $B$, the corresponding force is $\mathbf{f}_{2}^{B}$ and the couple $\mathbf{c}^{B}$, in the same sense as $\mathbf{c}^{A}$ because now the only contribution to the couple comes from $\mathbf{f}_{2}^{B}$.

momentum equation mentioned in Sec. II.) In Stokes flow this term can be evaluated with the result

$$
\Delta_{i j k}=-\beta \mu \epsilon_{i k a}\left[\frac{8}{5} \epsilon_{a j l}\left(u_{l}-w_{l}\right)+a^{2} \partial_{j}(\boldsymbol{\nabla} \times \mathbf{u})_{a}\right] .
$$

Here $\mathbf{u}$ and $u_{l}$ denote the part of the fluid velocity regular at the center of the particle which, at lowest order in the volume fraction, can be taken equal to the average volumetric flux $\mathbf{u}_{m}$.

\section{PHYSICAL INTERPRETATION}

The physical origin of the antisymmetric contribution to the mixture stress tensor can be clarified by considering the mesoscopic volume element shown in Fig. 5. Let us focus on particles such as $A$ and $B$ straddling the surface and let $\mathbf{F}$ be the resultant of the external body forces acting on particle material; for simplicity we draw this and other forces parallel to the face of the volume element. With the neglect of inertia, for each particle, this force is balanced by a hydrodynamic force $\mathbf{f}$. We divide this total hydrodynamic force in two parts, $\mathbf{f}_{1}$ and $\mathbf{f}_{2}$, the resultants of the tractions acting, respectively, on the portion of the particle surface inside and outside the volume element. These forces are uniquely and unambigu-

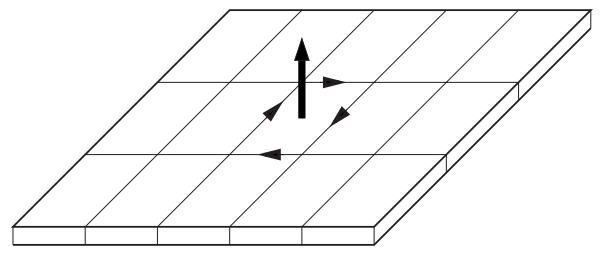

FIG. 6. The central tile represents the volume element at the top of the previous figure. The imbalance in the strength of the couples transmitted across the particles straddling the surfaces bounding the volume element is equivalent to the couple denoted by the bold vector.

ously defined, and so are their points of application (which, in general, will not be at the particle center). In keeping with Cauchy's stress principle, we are interested in the force and couple acting across the dashed line which demarcates the boundary of the control volume. For this purpose, according to a well-known statics theorem, the action of forces $\mathbf{F}$ and $\mathbf{f}_{2}$ for particle A and force $\mathbf{f}_{2}$ for particle $\mathrm{B}$ can be replaced by forces $\mathbf{F}-\mathbf{f}_{2}$ and $\mathbf{f}_{2}$, respectively, acting as shown in the lowest part of the figure, plus suitable couples $\mathbf{c}$ (which are of course balanced by equal couples due to $\mathbf{f}_{1}$ for particle $\mathrm{A}$ and to $\mathbf{F}$ and $\mathbf{f}_{1}$ for particle B). As indicated in the figure, the two couples act in the same sense.

The sum of all the forces $\mathbf{F}-\mathbf{f}_{2}$ for particles such as A and $\mathbf{f}_{2}$ for particles such as $B$ is the particle contribution to the mixture stress. Likewise, the sum of all the couples leads to $\mathbf{C} \cdot \mathbf{N}$, the dot product of the couple flux (30) into the local normal. For a uniform system, the total couple flux, i.e., the integral of $\mathbf{C} \cdot \mathbf{N}$ over the surface of the mesoscopic volume element, vanishes as the effects of the couples acting on opposite pairs of faces balance. It is only if there is an imbalance in the strength of these couples-caused, e.g., by a different number of particles or by the action of different external forces-that a net effect would survive.

Pursuing this idea, it is seen, e.g., from Eq. (26), that the contribution of the vector $\mathbf{V}$ to the antisymmetric stress through its curl is equivalent to the presence of "effective" couples $[-(1 / 2) n \mathbf{L}]_{\text {eff }}$ acting on the volume element of the mixture. The way in which this equivalence arises is sketched in Fig. 6 in which the central tile represents the mesoscopic volume element shown at the top of the previous figure. If the particles are not homogeneous, as in the situation considered in Ref. 9, couples similar to c above also arise but through a very different physical mechanism.

Our derivation has been based on considering a surface cutting through the particles. In this connection the reader may refer to Batchelor's paper, ${ }^{7}$ and in particular, to the text surrounding the quotation given shortly after Eq. (5) above (see also Refs. 28, 30, and 31). The point here is that a consistent mental picture of the homogenized system must be based on an "average" effective continuum, rather than on a single realization of the original disperse system. This "average" effective continuum must be such that the same fraction of any macroscopically small volume or surface element is occupied by the disperse phase. This requirement is widely appreciated in the literature and has given rise (see, e.g., Refs. 35 and 36) to the standard notion of representative elementary volume of volume averaging. ${ }^{37}$ 


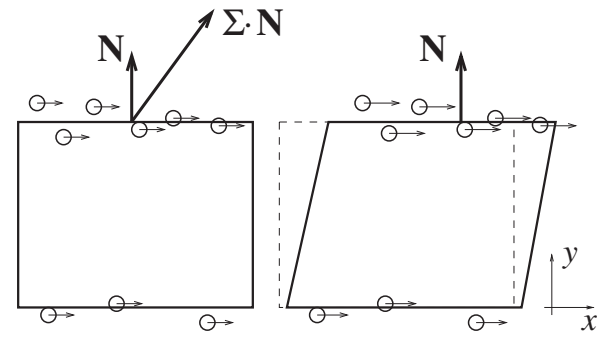

FIG. 7. Qualitative explanation of the physical origin of the new contributions to the symmetric stress shown in Eq. (40). Let, e.g., the external force on the particles straddling the upper surface be greater than that on the particles in the lower surface. The deformation of the volume element will be aided or, in other words, a smaller average shear stress needs to be applied to the faces of the volume element to obtain a given deformation. The same conclusion would be reached if the number of particles straddling the upper surface were greater than for the lower surface.

\section{SYMMETRIC STRESS}

The previous considerations suggest that spatial nonuniformity could also affect the symmetric part of the stress, which is responsible for the deformation of the volume element. Indeed, suppose that the external forces or the number of particles straddling the upper surface are greater than for the lower surface (Fig. 7). It is intuitively clear that, in this case, the deformation of the volume element will be aided or, in other words, that a smaller average shear stress needs to be applied to the faces of the volume element to obtain a given deformation.

For the case of Stokes flow, the analysis summarized in Appendix D leads to the conclusion that, in pure shear, the deviatoric part $\left\langle\tau_{x y}\right\rangle^{S}$ of the symmetric mixture stress has the form

$$
\left\langle\tau_{x y}\right\rangle^{S} \simeq \mu_{\mathrm{eff}} \frac{\partial u_{m}}{\partial y}-\mu_{\Delta} \frac{\partial}{\partial y}\left(\bar{w}-u_{m}\right)-\mu_{\nabla}\left(\bar{w}-u_{m}\right) \frac{\partial \beta}{\partial y} .
$$

Here $\mu_{\text {eff }}$ is the usual effective viscosity of the suspension, while $\mu_{\Delta}$ and $\mu_{\nabla}$ are new (positive) viscosity parameters. The minus signs account for the decrease in the shear force $\left\langle\tau_{x y}\right\rangle^{S}$ necessary to overcome the viscous resistance $\mu_{\text {eff }}\left(\partial u_{m} / \partial y\right)$ to the deformation. The relative velocity $\bar{w}-u_{m}$ might arise due to the action of an external force acting on the particles, inertia, or to other causes, such as spatial nonuniformities of the flow or of the particle distribution.

The importance of terms proportional to $\nabla \beta$ and $\boldsymbol{\nabla}\left(\bar{w}-u_{m}\right)$ for the stability of fluidized beds has been stressed, among others, by Batchelor in Ref. 38. Our analysis points to the existence of one possible mechanism giving rise to such terms.

In a nonuniform Stokes mixture, in tensor form, the deviatoric part of the viscous stress (40) is

$$
\langle\boldsymbol{\tau}\rangle^{S}=2 \mu_{\mathrm{eff}} \mathrm{E}_{m}-2 \mu_{\Delta} \mathrm{E}_{\Delta}-2 \mu_{\nabla} \mathrm{E}_{\nabla},
$$

in which $\mathrm{E}_{m}$ is the rate of deformation of the mixture volumetric flux, $E_{\Delta}$ is the analogous quantity for the relative velocity $\overline{\mathbf{w}}-\mathbf{u}_{m}$ :

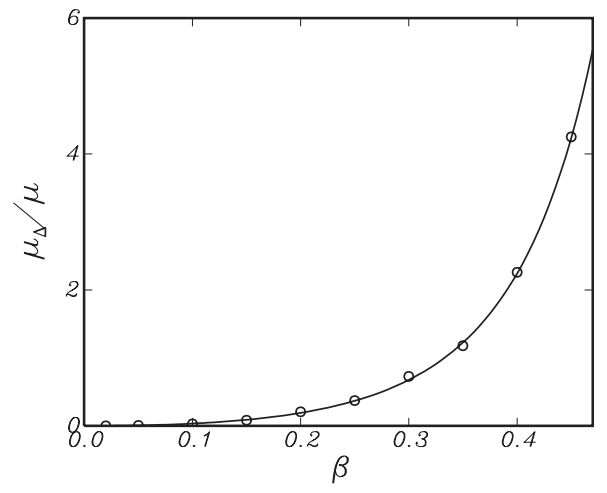

FIG. 8. Volume fraction dependence of the dimensionless viscosity parameter $\mu_{\Delta} / \mu$ appearing in Eq. (41). The solid line is the fit (44) and the symbols the numerical results found as described in Appendix D.

$$
2 \mathrm{E}_{\Delta}=\boldsymbol{\nabla}\left(\overline{\mathbf{w}}-\mathbf{u}_{m}\right)+\boldsymbol{\nabla}\left(\overline{\mathbf{w}}-\mathbf{u}_{m}\right)^{T}-\frac{2}{3}\left[\boldsymbol{\nabla} \cdot\left(\overline{\mathbf{w}}-\mathbf{u}_{m}\right)\right] \mathbf{I}
$$

and

$$
\begin{aligned}
2 \mathrm{E}_{\nabla}= & \left(\overline{\mathbf{w}}-\mathbf{u}_{m}\right) \boldsymbol{\nabla} \beta+(\boldsymbol{\nabla} \beta)\left(\overline{\mathbf{w}}-\mathbf{u}_{m}\right) \\
& -\frac{2}{3}\left[(\boldsymbol{\nabla} \beta) \cdot\left(\overline{\mathbf{w}}-\mathbf{u}_{m}\right)\right] \mathbf{I},
\end{aligned}
$$

in which $\mathbf{I}$ is the identity two-tensor; the viscosity parameters $\mu_{\Delta}$ and $\mu_{\nabla}$ can be represented as

$$
\begin{aligned}
\frac{\mu_{\Delta}}{\mu} & =\frac{2.7 \beta^{2}}{\left(1-\beta / \beta^{*}\right)^{1.57+1.80 \beta}}, \\
\frac{\mu_{\nabla}}{\mu} & =\frac{7.5 \beta}{\left(1-\beta / \beta^{*}\right)^{3.77-1.28 \beta}},
\end{aligned}
$$

with $\beta^{*}=0.78$. These relations are graphed in Figs. 8 and 9. The conventional effective viscosity may be represented in the form (see, e.g., Ref. 39)

$$
\mu_{\mathrm{eff}} / \mu=\left(1-\beta / \beta^{*}\right)^{-2} .
$$

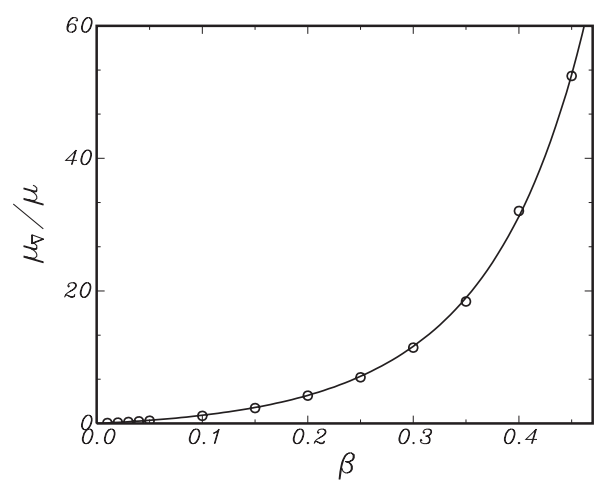

FIG. 9. Volume fraction dependence of the dimensionless viscosity parameter $\mu_{\nabla} / \mu$ appearing in Eq. (41). The solid line is the fit (45) and the symbols the numerical results found as described in Appendix D. 


\section{NUMERICAL EXAMPLE}

In order to show the quantitative importance of the present results for the stress, we carried out numerical simulations based on a simple mixture model with negligible inertia forces. If both phases are incompressible, the volumetric flux is divergenceless:

$$
\boldsymbol{\nabla} \cdot \mathbf{u}_{m}=0 .
$$

The linearized total mixture momentum equation takes the form $^{40}$

$$
\rho_{m} \frac{\partial \mathbf{u}_{m}}{\partial t}=-\nabla p_{m}+\nabla \cdot\langle\boldsymbol{\tau}\rangle^{S}-\nabla \times \nabla \times \mathbf{V}+\rho_{m} \mathbf{g},
$$

in which $\langle\boldsymbol{\tau}\rangle^{S}$ is the deviatoric part of the symmetric stress and $\rho_{m}$ is the mean mixture density. We have retained the time derivative, even if small, in order to be able to adopt a straightforward numerical method. ${ }^{41}$ After multiplication by the particle volume, the relation expressing the conservation of the particle number becomes

$$
\frac{\partial \beta}{\partial t}+\nabla \cdot(\beta \overline{\mathbf{w}})=0
$$

Shear-induced diffusion is not included in this equation as the only models available are for parallel flow. In any event, our purpose here is only to demonstrate the differences due to the various mixture stress models. The average particle velocity $\overline{\mathbf{w}}$ follows from the quasistatic balance of forces and may be written as

$$
\overline{\mathbf{w}}=\mathbf{u}_{m}+H(\beta) \mathbf{w}_{s},
$$

in which $\mathbf{w}_{s}$ is the (constant) settling velocity of a single particle.

In general, the issue of boundary conditions in simulations of this type is a nontrivial one (for a related problem see, e.g., Ref. 42). We use no-slip on both $\mathbf{u}_{m}$ and $\overline{\mathbf{w}}$, which is equivalent to no-slip on the pure fluid velocity field as the particles never reach the wall in the present simulation.

We apply the previous model to the two-dimensional gravitational settling of an initially cylindrical mixture "blob" in a container of width $L$ and height $8 L$ filled with pure fluid. We nondimensionalize the equations in terms of $\rho^{F}$, $g$, and $L$ and take $\rho^{P} / \rho^{F}=3, a / L=0.07$ (with $a$ the particle radius) and $\left|\mathbf{w}_{s}\right| / \sqrt{g L}=0.2178, \nu^{F} / \sqrt{L^{3} g}=0.1$, in which $\nu^{F}$ is the fluid kinematic viscosity. The Reynolds number estimated as $(1 / 2) L\left|\mathbf{w}_{s}\right| / \nu^{F}$ equals 1.09 ; the actual value is however smaller in view of the hindrance effect of the particles and the wall, which decreases the velocity.

The numerical method is a simple adaptation of the standard first-order projection procedure. We define

$$
\mathbf{u}^{*}=\mathbf{u}^{n}+\frac{\Delta t}{\rho_{m}}\left[\boldsymbol{\nabla} \cdot\langle\boldsymbol{\tau}\rangle^{S}-\boldsymbol{\nabla} \times \boldsymbol{\nabla} \times \mathbf{V}\right]^{n}+\Delta t \mathbf{g},
$$

where the superscript $n$ denotes values at time level $t^{n}$ and $\Delta t$ is the time step. The condition (47) of global mixture incompressibility gives

$$
\boldsymbol{\nabla} \cdot\left(\frac{1}{\rho_{m}^{n}} \boldsymbol{\nabla} p_{m}^{n+1}\right)=\frac{\boldsymbol{\nabla} \cdot \mathbf{u}^{*}}{\Delta t}
$$

which is solved by iteration and determines $p_{m}^{n+1}$. The new velocity field is then obtained from

$$
\mathbf{u}_{m}^{n+1}=\mathbf{u}^{*}-\frac{\Delta t}{\rho_{m}} \boldsymbol{\nabla} p_{m}^{n+1} .
$$

Once $\mathbf{u}_{m}$ is known, $\overline{\mathbf{w}}$ can be calculated from Eq. (50) as

$$
\overline{\mathbf{w}}^{n+1}=\mathbf{u}_{m}^{n+1}+H\left(\beta^{n}\right) \mathbf{w}_{s},
$$

and the particle volume fraction updated from Eq. (49) according to

$$
\beta^{n+1}=\beta^{n}-\Delta t \boldsymbol{\nabla} \cdot\left(\beta^{n} \overline{\mathbf{w}}^{n+1}\right) .
$$

The spatial operators were approximated by central differences on a staggered grid, except in the volume fraction equation (55) which was discretized with the so-called Superbee flux limiter (see, e.g., Ref. 43). A standard grid refinement test showed that a mesh length equal to $L / 32$ gave converged results.

We ran several simulations of the same basic process changing the form used for the symmetric part of the viscous stress $\langle\boldsymbol{\tau}\rangle^{S}$. Figure 10 shows the evolution of the system at different times together with the instantaneous streamlines when the conventional form $\langle\boldsymbol{\tau}\rangle^{S}=\mu_{\mathrm{eff}}\left(\boldsymbol{\nabla} \mathbf{u}_{m}+\boldsymbol{\nabla} \mathbf{u}_{m}^{T}\right)$ is used. The gray scale (color online) indicates the particle volume fraction. The particles near the downward-facing edge of the blob fall gradually faster as the local concentration decreases. This process causes a depletion of the outer layers and, by conservation of mass, an inwardly directed flow, which compresses the blob laterally. The result is an elongation of the structure and an apparent diffusive behavior around its edges. The falling blob gives rise to a recirculating flow which, in its turn, generates countervortices near the top and bottom walls.

The effect of the new terms added to the viscous stress is illustrated for this case in Fig. 11. The leftmost panel is the result of the conventional stress model from the previous figure. The second panel is the result of our new model. The blob is seen to fall faster and the isolines of constant $\beta$ are also deformed. The next three panels show the individual effects of the new terms in the stress added to the conventional model. In the order in which they appear in the figure, they are the antisymmetric component, the term proportional to $\mu_{\Delta}$ and the term proportional to $\mu_{\nabla}$ in Eq. (41). The antisymmetric stress by itself is seen to slightly retard the fall as does, if to a somewhat smaller extent, the $\mu_{\Delta}$ term. In this particular example, the strongest effect is found for the $\mu_{\nabla}$ term which contains the relatively large volume fraction gradients. It may also be noted that, due to the decrease in the hindrance function with $\beta, \nabla \beta$ and $\boldsymbol{\nabla}\left(\overline{\mathbf{w}}-\mathbf{u}_{m}\right)$ have opposite signs and therefore tend to oppose each other. The difference between the models accumulates with time and would be greater than in this simple example in situations such as, e.g., longer falls, stronger gradients, different initial particle distribution, and others. 

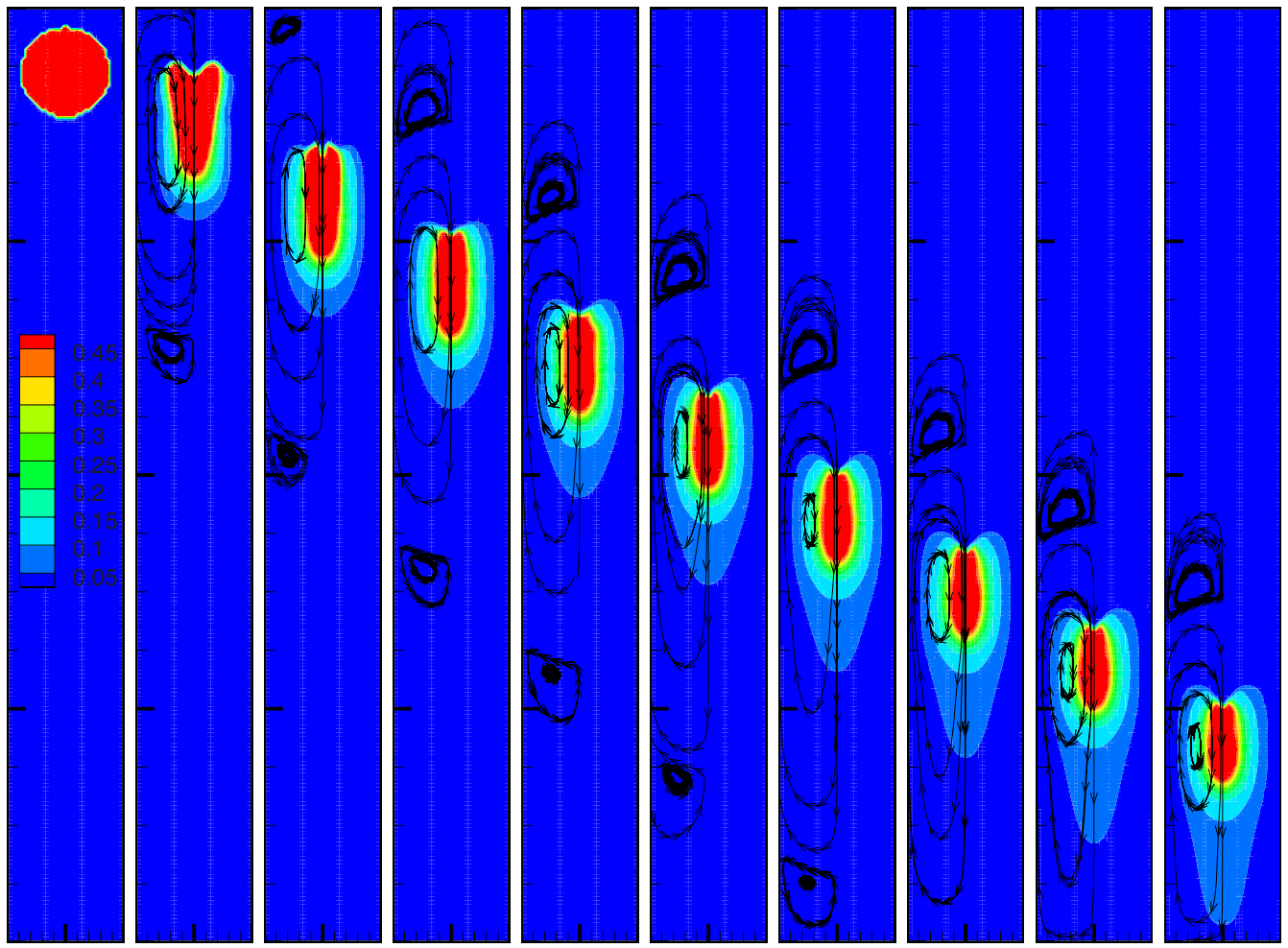

FIG. 10. (Color online) Volume fraction distribution (gray scale, from 0.05 to 0.45 ) at different instants in the two-dimensional gravitational settling of a mixture "blob" with the standard purely symmetric stress expressed in terms of an effective viscosity. The lines are the instantaneous streamlines. The panels shown are at times $t \sqrt{g / L}=0,40,80,120,160,200,240,280,320$, and 360 .

The literature contains several studies of sedimenting particle clouds in unbounded fluids (see, e.g., Refs. 44-46), which, however, differ from the present situation due to the dimensionality (two versus three dimensions) and the dominant effect of the lateral boundaries. Vortical structures on the two sides of the blob are also found in those studies but, here, their center is pushed outward due to the large viscosity at its core dense with particles. The lateral walls elongate these structures so that the upper stagnation point on the axis is removed and, with it, the leakage of particles from the back of the blob.

\section{POTENTIAL FLOW}

In the case of potential flow the vector $\mathbf{V}$ vanishes, as is evident from Eq. (30). Omitting the contribution of the external couples, the antisymmetric component of the stress reduces to the two $\Delta$ terms. In this particular case it is readily found that

$$
\Delta_{p k q}=\frac{1}{5} n a^{2}\left(\delta_{p q}\left\langle\oint p n_{k} \mathrm{~d} S\right\rangle-\delta_{p k}\left\langle\oint p n_{q} \mathrm{~d} S\right\rangle\right) .
$$

Up to a sign, the integrals equal the hydrodynamic force on the particle. As noted before in Sec. V, this term has no dynamical consequences as far as the average momentum equation is concerned and we will not consider it further.

By using Batchelor's result (4.5) for the integral in Eq. (10), we find

$$
(1-\beta)\left\langle\boldsymbol{\sigma}^{F}\right\rangle+\beta\left\langle\boldsymbol{\sigma}^{P}\right\rangle=-(1-\beta)\langle p\rangle \mathbf{I}-n a\langle\oint p \mathbf{n n d} S\rangle .
$$

Since repeated averaging has no effect on an averaged quantity, this expression can also be written as

$$
\begin{aligned}
& (1-\beta)\left\langle\boldsymbol{\sigma}^{F}\right\rangle+\beta\left\langle\boldsymbol{\sigma}^{P}\right\rangle \\
& =-\langle p\rangle \mathbf{I}-n a\langle\oint(p-\langle p\rangle) \mathbf{n n d} S\rangle .
\end{aligned}
$$

Upon taking the divergence to form the momentum equation, the first term is just the gradient of the mean pressure. The second term is an additional contribution to the stress which has been identified by several authors, if in slightly different though equivalent form. If we write

$$
\begin{aligned}
& \langle\oint(p-\langle p\rangle) \mathbf{n n d} S\rangle \\
& =\left\langle\oint p\left(\mathbf{n n}-\frac{1}{3} \mathbf{I}\right) \mathrm{d} S\right\rangle+\frac{1}{3}\langle\oint(p-\langle p\rangle) \mathrm{d} S\rangle \mathbf{I},
\end{aligned}
$$

we recover the form given in Ref. 24. In the same reference it is shown that this result coincides with that of Ref. 21 and can also be reconciled with that proposed on heuristic grounds in Ref. 20. To the first order in $\beta$, it is shown in Ref. 24 that 

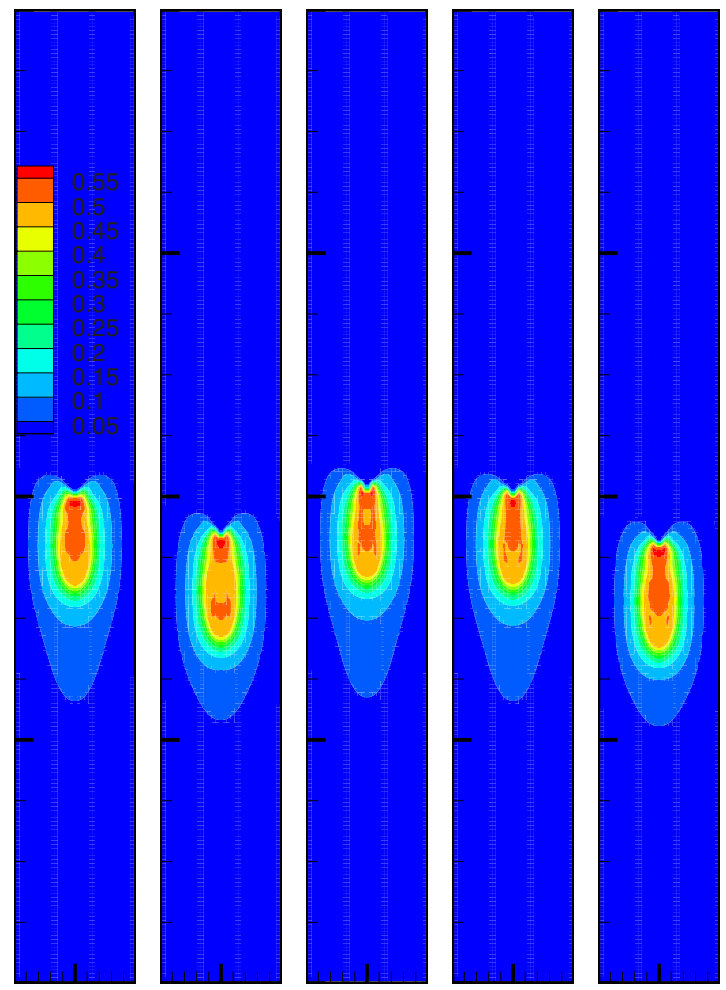

FIG. 11. (Color online) Volume fraction distribution (gray scale, from 0.05 to 0.55 ) as predicted by the different models for the gravitational settling problem of the previous figure at $t \sqrt{g / L}=240$. The leftmost panel is the result of the conventional stress model from the previous figure. The second panel is the result of our complete new model with antisymmetric stress and augmented symmetric stress. The next three panels show the individual effects of the new terms in the stress added to the conventional model. In the order in which they appear in the figure, they are the antisymmetric component, the term proportional to $\mu_{\Delta}$ and the term proportional to $\mu_{\nabla}$ in Eq. (41).

$$
\begin{aligned}
n a & \langle\oint(p-\langle p\rangle) \mathbf{n n d} S\rangle \\
& =\frac{1}{5} \beta \rho^{F}\left[2\left|\mathbf{u}_{m}-\overline{\mathbf{w}}\right|^{2} \mathbf{I}-\frac{9}{4}\left(\mathbf{u}_{m}-\overline{\mathbf{w}}\right)\left(\mathbf{u}_{m}-\overline{\mathbf{w}}\right)\right],
\end{aligned}
$$

up to terms containing the particle Reynolds stress.

It is interesting to compare Eq. (59) with another form for the particle stress that appears in the literature. ${ }^{21,26} \mathrm{In}$ Ref. 26, it is found that the average momentum equation for the particles may be written as

$$
\overline{n\left(m^{P} \dot{\mathbf{w}}+\dot{\mathbf{J}}\right)}=\boldsymbol{\nabla} \cdot\left[\left\langle(1-\chi) \mathbf{M}^{F}\right\rangle+n\left\langle\oint \mathbf{r M}^{F} \cdot \mathbf{n} \mathrm{d} S\right\rangle\right] .
$$

Here $\mathbf{J}$ is the hydrodynamic impulse defined by

$$
\mathbf{J}=-\rho^{F} \oint \phi \mathbf{n} \mathrm{d} S,
$$

in which $\phi$ is the velocity potential (see, e.g., Ref. 47), $\chi$ is the characteristic function of the particle phase and the tensor $\mathrm{M}$ is given by

$$
\mathbf{M}^{F}=\frac{1}{2}\left(\mathbf{u}^{F} \cdot \mathbf{u}^{F}\right) \mathbf{I}-\mathbf{u}^{F} \mathbf{u}^{F} .
$$

Equation (61) can be interpreted as the average equation of motion of fictitious particles with an apparent momentum $m^{P} \mathbf{w}+\mathbf{J}$. The last term bears a striking similarity to the quantity $\oint \mathbf{r}\left(\boldsymbol{\sigma}^{P} \cdot \mathbf{n}\right) \mathrm{d} S$ arising in Batchelor's analysis [Eq. (4.6) in Ref. 7] and it is interesting to understand it from this point of view. In so doing, we will also be led to a much simpler derivation of the result (61).

Let us consider a fictitious system governed, outside a set of $N$ equal spheres, by

$$
\boldsymbol{\nabla} \cdot \mathrm{M}^{F}=0 .
$$

This is formally the same as the momentum equation for an inertia-less fluid. Inside the spheres, we assume

$$
\left(m^{P} \dot{\mathbf{w}}+\dot{\mathbf{J}}\right) \delta\left(\mathbf{x}-\mathbf{y}^{\alpha}\right)=\boldsymbol{\nabla} \cdot \mathbf{M}^{P} \quad\left|\mathbf{x}-\mathbf{y}^{\alpha}\right| \leq a, \quad 1 \leq \alpha \leq N,
$$

where the $\delta$ function signifies that the inertia has been localized at the particle center. On the surface of each sphere, we impose continuity of the normal stress, $\left(\mathrm{M}^{F}-\mathrm{M}^{P}\right) \cdot \mathbf{n}=0$. (As stated the problem is insufficiently specified in a mathematical sense as there are more unknowns than equations. One may imagine adding other constraints which have no consequences for the purpose of this argument.) Due to this condition, the average momentum equation for the entire system is

$$
n\left\langle m^{P} \dot{\mathbf{w}}+\mathbf{J}\right\rangle=n \overline{\left(m^{P} \dot{\mathbf{w}}+\dot{\mathbf{J}}\right)}=\boldsymbol{\nabla} \cdot\left\langle(1-\chi) \mathrm{M}^{F}+\chi \mathrm{M}^{P}\right\rangle,
$$

where the first step follows from the fact that the entire inertia of each sphere is concentrated at its center. The lefthand side has the appearance of a particle momentum equation although, since the fluid has negligible inertia, this is in fact a momentum balance for the entire mixture. If the quantity in the right-hand side is interpreted as a Cauchy stress, the same argument used before to derive Eq. (10) leads to the result

$$
\beta\left\langle\mathrm{M}^{P}\right\rangle=n\left\langle\int \mathrm{M}^{P} \mathrm{~d} v\right\rangle=n\left\langle\oint \mathbf{r}\left(\mathrm{M}^{F} \cdot \mathbf{n}\right) \mathrm{d} S\right\rangle,
$$

from which Eq. (61) follows.

We conclude that, if the virtual mass contribution is left as a piece of the hydrodynamic force, the particle contribution to the stress takes the form $\left\langle-\int p \operatorname{rnd} S\right\rangle$ while, if it is considered as a part of the apparent momentum of the particles, the particle contribution to the stress is $\left\langle\int \mathbf{r}\left(\mathrm{M}^{F} \cdot \mathbf{n}\right) \mathrm{d} S\right\rangle$.

Earlier papers ${ }^{21,26}$ refer to the quantity under the divergence sign in Eq. (61) as the "particle stress," which is seen to be the stress that would arise in the fictitious medium framework just discussed.

\section{DISCUSSION}

Our analysis, based on the Cauchy concept of stress, has identified circumstances under which the mesoscale average stress in a disperse system is not symmetric. In the first place, this may happen in the presence of external couples 


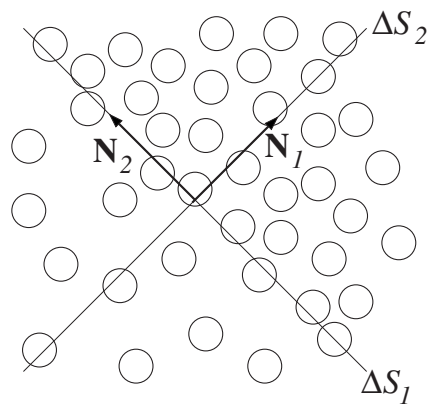

FIG. 12. Illustration of the mechanism responsible for the loss of symmetry of the average stress tensor. Symmetry would require that $\mathbf{N}_{2} \cdot\left(\boldsymbol{\Sigma} \cdot \mathbf{N}_{1}\right)$ $=\mathbf{N}_{1} \cdot\left(\boldsymbol{\Sigma} \cdot \mathbf{N}_{2}\right)$. In the presence of nonuniformities, e.g., in the particle concentration as sketched here, the total force transmitted across the surface elements $\Delta S_{1}$ and $\Delta S_{2}$ will be different.

applied to the particles, as found by Batchelor $^{7}$ and Brenner. ${ }^{17,48}$ But the average stress of hydrodynamic origin may be nonsymmetric also in the presence of spatial nonuniformities of the particle number density or other particle quantities. ${ }^{4}$ The root of this effect in nonzero spatial gradients explains why it was not encountered in most of the existing studies.

The mechanism by which the average of microscopically symmetric stresses gives rise to a mesoscopic nonsymmetric quantity can be illustrated with reference to Fig. 12. This figure shows two macroscopic surface elements with orthogonal normals in a nonuniform mixture. There is no reason to expect that, if the particles are subjected to an external force, the net force in direction 2 transmitted through the particles cut across by surface element 1 should equal the net force in direction 1 transmitted through surface element 2. In other words, $\mathbf{N}_{2} \cdot \boldsymbol{\Sigma} \cdot \mathbf{N}_{1} \neq \mathbf{N}_{1} \cdot \boldsymbol{\Sigma} \cdot \mathbf{N}_{2}$.

Of course, if one were to take the limit $a \rightarrow 0$ keeping the number density constant, the effect would disappear. But if the limit is taken keeping constant the particle volume fraction, the number density grows indefinitely and the same picture of Fig. 12 would apply independent of how small $a$ is made. This is the proper limit to take in an average equation framework, just as in the case of a real gas or liquid described as a continuum.

Mathematically, the operator that interchanges the two indices of the macroscopic stress tensor interchanges also the surface elements and is therefore different from the operator that interchanges the two indices of the microscopic stress tensor. In other words, averaging and index interchange do not commute at the mesoscale (although, of course, they do at the microscale: the ensemble average stress at a geometric point is symmetric). Thus, lack of symmetry is a feature which emerges at the mesoscale. The situation has some similarity with the loss of time reversibility encountered with the Boltzmann equation, which describes the evolution of a system at intermediate, "coarse-grained" time scales, longer than molecular times (over which the evolution is timereversible), but shorter than macroscopic times (over which collisions are not even recognized).

We can explain how the present results relate to the equivalent ones obtained by ensemble averaging in the fol- lowing way. The ensemble-averaged particle contribution to the stress, $\overline{\mathbf{s}}^{P}$, may be expanded in a multipole series (see, e.g., Refs. 49 and 50)

$$
\begin{aligned}
\bar{s}_{i j}^{P}= & n \overline{\oint r_{j}(\boldsymbol{\sigma} \cdot \mathbf{n})_{i} \mathrm{~d} S}-\frac{1}{2} \boldsymbol{\nabla} \cdot\left[\overline{\oint r_{j}(\boldsymbol{\sigma} \cdot \mathbf{n})_{i} \mathbf{r} \mathrm{d} S}\right] \\
& +\frac{1}{3 !} \boldsymbol{\nabla} \boldsymbol{\nabla}:\left[\overline{\hat{\oint} r_{j}(\boldsymbol{\sigma} \cdot \mathbf{n})_{i} \mathbf{r r} \mathrm{d} S}\right]+\ldots,
\end{aligned}
$$

where the overline denotes the ensemble average. The symmetric part of the first term scales like $n a^{3} \mu \nabla u_{m}=\beta \mu \nabla u_{m}$ multiplied by a function of $\beta$. The second term is the one giving rise to the antisymmetric stress and has been shown in Sec. $\mathrm{V}$ to be of the order of $\beta \mu \nabla \times\left(\bar{w}-u_{m}\right)$. For dimensional reasons, all the other terms in the multipole expansion must be multiplied by a power of $a$ sufficient to balance the inverse length of the gradient operators. Therefore, in order of magnitude and aside from functions of $\beta$ of order of one, the expansion (68) is like

$$
\begin{aligned}
\overline{\mathrm{s}}^{P} \sim & \beta \mu \nabla u_{m}+\beta \mu \nabla \times\left(\bar{w}-u_{m}\right) \\
& +\frac{a}{L} \beta\left[O(1)+O\left(\frac{a}{L}\right)+O\left(\frac{a^{2}}{L^{2}}\right)+\ldots\right] .
\end{aligned}
$$

If all the terms of the series are retained, the result is the exact microscopic ensemble average stress, which is symmetric. If, on the other hand, one takes the continuum limit $a / L \rightarrow 0$ for constant $\beta$ before summing the series, one is left with an approximation to the stress, which can rightly be labeled mesoscopic, which is not symmetric. This is the procedure followed in our earlier paper. ${ }^{5}$

Bardet and Verdoulakis ${ }^{51}$ have calculated the stress in a granular medium by applying the principle of virtual work and found it to have an antisymmetric component when the forces on the particles have nonzero moment about their centers. This is similar to our result (20) and, in fact, their Eq. (47) is quite analogous to our result (24). [Their moments at contact, $m_{i}^{e}$, are analogous to our applied couples $L_{i}$ and, in their situation, the couple $\mathbf{r} \times\left(\boldsymbol{\sigma}^{P} \cdot \mathbf{N}\right)$ is only applied at the particle surface.] The same authors however find a symmetric stress if they calculate it via a volume average under assumption of uniformity.

The recent homogenization analysis of Ref. 52 shows that, when the applied loading on a dilute elastic composite is nonuniform, effects which can be approximately accounted for by a Cosserat (i.e., micropolar) model arise. The appendix of this work presents an interesting and concise overview of the controversies related to Cosserat effects in elastic composites. However, the authors find that the Cosserat model cannot reproduce the exact result of the homogenization, which suggests that it does not account precisely for the relevant physics. On the other hand, very recent work $^{53}$ finds experimental evidence for such effects.

The results presented in Sec. III, in which the system is assumed to be locally uniform, fail to predict an antisymmetric component, while the analysis of Appendix $\mathrm{C}$ for a weakly nonuniform system does lead to such a component intimately connected to the lack of spatial homogeneity. Similarly, the antisymmetric component identified in Ref. 51 
only arises from the boundary of the medium, which is the only region where a nonuniformity is present in their otherwise uniform system. Lack of uniformity appears therefore to be essential in causing the lack of symmetry of the mixture stress. This remark might explain the conflicting results found by various authors.

One of the specific examples we have presented in Secs. V, VII, and VIII has been based on closure relations obtained for the hard-sphere distribution function. It is well-known that the particle distribution function is actually flow dependent (see, e.g., Refs. 54-58) and, therefore, our results should only be taken as an illustrative example. Furthermore, the symmetric stress in our example does not contain other non-Newtonian effects such as normal stress differences, which are also due to flow-dependent particle distribution (see, e.g., Refs. 56 and 59-61). However, the physical arguments that we have presented suggest that the mechanisms giving rise to the effects that we have identified should be present to some degree whatever the particle distribution function.

\section{CONCLUSIONS}

In this paper, we have shown how an elementary application of Cauchy's stress principle to the linear momentum balance of a disperse fluid-particle mixture, coupled with volume averaging, permits one to recover well-known expressions for the symmetric stress in a disperse system. The novel aspect of the analysis is that the same argument applied to the angular momentum balance points to the possible existence of a mesoscale antisymmetric component of the stress of hydrodynamic origin in the presence of spatial nonuniformities, e.g., in the particle concentration.

For purposes of illustration, we have applied the general results to several situations with and without inertia. In the former case, we have shown that the coefficient of the antisymmetric stress component increases proportionally to the square root of the particle Reynolds number, at least up to $\mathrm{Re}=50$ (Fig. 4).

In the absence of couples acting on the particles, the antisymmetric stress component vanishes for a spatially uniform system. Similar conclusions have been derived in the recent solid mechanics literature (e.g., Refs. 51 and 52), where the presence of so-called Cosserat effects has been a contentious point for some time. Our results (and especially those presented in Appendix A) suggest that such effects only arise in the presence of inhomogeneities, which are not incorporated in most analyses based on volume averaging.

\section{ACKNOWLEDGMENTS}

We are grateful to Dr. Hanneke Bluemink, Department of Applied Sciences, University of Twente, The Netherlands, for the calculation of the PHYSALIS results of Sec. V. The derivation of Eq. (A5) in Appendix A is patterned after an argument given in an unpublished note by Professor Leon van Dommelen, FAMU-FSU College of Engineering Tallahassee, Florida 32303, USA. The reviewers were very helpful with their probing questions, constructive suggestions and references to the solid mechanics literature.
This study was supported by NSF Grant Nos. CBET 0625138 and CBET 0754344.

\section{APPENDIX A: INTERPARTICLE FORCES}

One reviewer suggested that we examine how interparticle (and/or colloidal) forces would affect the results of this paper. It is well-known that such forces give a contribution to the stress given, in the present notation, by $-n\langle\mathbf{b y}\rangle$, where $\mathbf{b}$ is the interparticle force. ${ }^{62-64} \mathrm{We}$ can derive this result by the same procedure used in Sec. III.

The total (direct) force per unit area $\boldsymbol{\Sigma}^{i p}$ that particles outside the surface element of Fig. 1 exert on the particles inside is the interparticle contribution to the stress that we need to calculate and it is given by

$$
\Delta \mathcal{S} \boldsymbol{\Sigma}_{i j}^{i p} N_{j}=\sum_{\alpha \in \mathcal{V}} \sum_{\beta \notin \mathcal{V}} b_{i}^{\beta \rightarrow \alpha} .
$$

In a uniform system this average is independent of the location of $\Delta \mathcal{S}$ in the direction of $\mathbf{N}$ and therefore, if we consider $N_{S}$ surfaces $\Delta \mathcal{S}_{k}$ uniformly distributed over a thickness equal to the range $R$ of the interparticle force, we find that also

$$
\Delta \mathcal{S} \boldsymbol{\Sigma}_{i j}^{i p} N_{j}=\frac{1}{N_{S}} \sum_{k} \sum_{\alpha \text { below }} \Delta S_{k} \beta \text { above } \Delta S_{k} b_{i}^{\beta \rightarrow \alpha} .
$$

The distance between particles $\alpha$ and $\beta$ is $\left(\mathbf{y}^{\beta}-\mathbf{y}^{\alpha}\right) \cdot \mathbf{N}$ and if the distance between surfaces is $\delta z$, assumed much smaller than $R$, there are $\left(\mathbf{y}^{\beta}-\mathbf{y}^{\alpha}\right) \cdot \mathbf{N} / \delta z$ planes separating the two particles. Furthermore, the restrictions that particle $\alpha$ be below and particle $\beta$ be above $\Delta \mathcal{S}_{k}$ can be removed by considering both arrangements and dividing by 2 . Thus, Eq. (A2) becomes

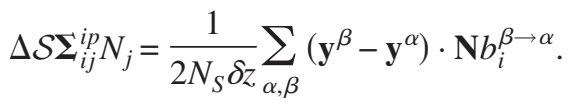

Now, we recognize that $N_{S} \delta z \Delta \mathcal{S}=R \Delta \mathcal{S}=\mathcal{V}$, the averaging volume (similar to the volume between dashed lines in Fig. 1 , but with a thickness $R$ rather than $2 a$ ) to find

$$
\Sigma_{i j}^{i p}=\frac{1}{2 \mathcal{V}} \sum_{\alpha, \beta} b_{i}^{\beta \rightarrow \alpha}\left(y_{j}^{\beta}-y_{j}^{\alpha}\right),
$$

which, upon interchange of $\alpha$ and $\beta$ noting that $b_{i}^{\beta \rightarrow \alpha}$ $=-b_{i}^{\alpha \rightarrow \beta}$, gives

$$
\Sigma_{i j}^{i p}=-\frac{1}{\mathcal{V}} \sum_{\alpha, \beta} b_{i}^{\beta \rightarrow \alpha} y_{j}^{\alpha}=-\frac{1}{\mathcal{V}} \sum_{\alpha} y_{j}^{\alpha} \sum_{\beta} b_{i}^{\beta \rightarrow \alpha}=-n\left\langle b_{i} y_{j}\right\rangle .
$$

In order to fully reconcile Eq. (10) with the results of Ref. 7 we use the identity

$$
\begin{aligned}
\int_{v} \boldsymbol{\sigma}^{P} \mathrm{~d} v & =\int_{v}\left[\boldsymbol{\nabla} \cdot\left(\mathbf{r} \boldsymbol{\sigma}^{P}\right)-\mathbf{r} \boldsymbol{\nabla} \cdot \boldsymbol{\sigma}^{P}\right] \mathrm{d} v \\
& =\oint \mathbf{r}\left(\boldsymbol{\sigma}^{P} \cdot \mathbf{n}\right) \mathrm{d} S-\rho^{P} \int_{v} \mathbf{r}\left(\mathbf{a}^{P}-\mathbf{g}\right) \mathrm{d} v,
\end{aligned}
$$

in which the last step follows from the momentum equation for the particle material 


$$
\rho^{P} \mathbf{a}^{P}=\boldsymbol{\nabla} \cdot \boldsymbol{\sigma}^{P}+\rho^{P} \mathbf{g},
$$

in which $\mathbf{g}$ is the body force. By using the continuity of normal stress at the particle surface, $\boldsymbol{\sigma}^{P}$ can be replaced by $\boldsymbol{\sigma}$, the stress in the fluid. ${ }^{65}$ Batchelor ${ }^{7}$ shows that the antisymmetric part of Eq. (A6) balances the external couple $L_{i}$ acting on the particle

$$
L_{i}=\epsilon_{i j k} \oint(\boldsymbol{\sigma} \cdot \mathbf{n})_{j} r_{k} \mathrm{~d} S-\epsilon_{i j k} \rho^{P} \int_{v}\left(a_{j}^{P}-b_{j}\right) r_{k} \mathrm{~d} v,
$$

so that, finally,

$$
\begin{aligned}
v\left\langle\boldsymbol{\sigma}^{P}\right\rangle= & \frac{1}{2}\left\langle\oint\left[\mathbf{r}(\boldsymbol{\sigma} \cdot \mathbf{n})+(\boldsymbol{\sigma} \cdot \mathbf{n}) \mathbf{r}-\frac{2}{3}(\mathbf{r} \cdot \boldsymbol{\sigma} \cdot \mathbf{n}) \mathbf{I}\right] \mathrm{d} S\right\rangle \\
& +\frac{1}{2}\left\langle\rho^{P} \int_{v}\left[\mathbf{r}\left(\mathbf{g}-\mathbf{a}^{P}\right)+\left(\mathbf{g}-\mathbf{a}^{P}\right) \mathbf{r}\right] \mathrm{d} v\right\rangle \\
& +\frac{1}{2} \boldsymbol{\epsilon} \cdot\langle\mathbf{L}\rangle+\frac{1}{3} \mathbf{I}\langle\oint \mathbf{r} \cdot \boldsymbol{\sigma} \cdot \mathbf{n} \mathrm{d} S\rangle .
\end{aligned}
$$

The first term is the average stresslet and the last one an isotropic contribution.

We now turn to the angular momentum balance of Sec. IV and in particular to the last term of Eq. (16) for which, in place of Eq. (17), we have

$$
\int_{v}(\mathbf{N} \cdot \mathbf{r}) \mathbf{r} \times\left(\boldsymbol{\nabla} \cdot \boldsymbol{\sigma}^{P}\right) \mathrm{d} v=\rho^{P} \int_{v}(\mathbf{N} \cdot \mathbf{r}) \mathbf{r} \times\left(\mathbf{a}^{P}-\mathbf{g}\right) \mathrm{d} v .
$$

For an essentially rigid, homogeneous particle the acceleration contribution can be evaluated as before finding, in place of Eq. (18),

$$
\begin{aligned}
& \rho^{P} \int_{v}(\mathbf{N} \cdot \mathbf{r}) \mathbf{r} \times\left(\mathbf{a}^{P}-\mathbf{g}\right) \mathrm{d} v \\
& \quad=\frac{1}{5} a^{2} m^{P} \mathbf{N} \times \dot{\mathbf{w}}-\rho^{P} \int_{v}(\mathbf{N} \cdot \mathbf{r}) \mathbf{r} \times \mathbf{g} \mathrm{d} v .
\end{aligned}
$$

Again using the particle equation of motion (A10) then becomes

$$
\begin{aligned}
\int_{v}(\mathbf{N} \cdot \mathbf{r}) \mathbf{r} \times\left(\boldsymbol{\nabla} \cdot \boldsymbol{\sigma}^{P}\right) \mathrm{d} v \\
=\frac{1}{5} a^{2} \mathbf{N} \times\left[\oint \boldsymbol{\sigma}^{F} \cdot \mathbf{n} \mathrm{d} S+\rho^{P} \int \mathbf{g} \mathrm{d} v\right] \\
\quad-\rho^{P} \int_{v}(\mathbf{N} \cdot \mathbf{r}) \mathbf{r} \times \mathbf{g} \mathrm{d} v .
\end{aligned}
$$

As a result, the expression (21) for the couple flux tensor C is augmented by the term

$$
\mathrm{C}_{i j}^{\prime}=n \epsilon_{i k l}\left\langle\rho^{P} \int\left(r_{j} r_{k}-\frac{1}{5} a^{2} \delta_{j k}\right) g_{l} \mathrm{~d} v\right\rangle .
$$

In particular, if $\mathbf{g}$ is uniform, $\mathrm{C}_{i j}^{\prime}=0$ and there is no contribution to the couple flux tensor and, therefore, to the antisymmetric stress.

\section{APPENDIX B: ANGULAR MOMENTUM BALANCE}

It was stated in Sec. IV that the mixture has no intrinsic angular momentum. If the particles can rotate, this assertion appears to be at variance with statements often encountered in the literature, and it is useful to clarify the situation here. A deeper understanding of the issue requires ensemble averaging, but the gist of the argument can also be described in the present context of volume averaging.

There are two ways to define the particle contribution to the average mixture velocity. In the first one, which is that adopted here, the particles contribute to the average the actual velocity at every point in their interior. For example, for rigid particles, we would write

$$
\left\langle\mathbf{u}^{P}\right\rangle=\langle\mathbf{w}+\boldsymbol{\Omega} \times \mathbf{r}\rangle,
$$

where $\mathbf{w}$ and $\boldsymbol{\Omega}$ are the translational and rotational velocities. Alternatively, one might include only the translational velocity writing

$$
\left\langle\left\langle\mathbf{u}^{P}\right\rangle\right\rangle=\langle\mathbf{w}\rangle .
$$

The difference between $\left\langle\mathbf{u}^{P}\right\rangle$ and $\left\langle\left\langle\mathbf{u}^{P}\right\rangle\right\rangle$ may be interpreted as the particle spin and considered as an intrinsic angular momentum. It is evident that the right-hand side of the angular momentum balance equation must be different according as the total angular momentum is calculated with respect to the average velocity (B1) or (B2). As long as one remains at a fundamental level, as we do in this paper, the definition (B1) seems simpler, as will be seen shortly. However, the second definition might be more useful, for example, if one attempts to develop approximate closure relations for some specific case.

With the definition (B1), the average linear momentum balance for the mixture can be written as

$$
\begin{aligned}
\frac{\partial}{\partial t}\left[(1-\beta) \rho^{F}\left\langle\mathbf{u}^{F}\right\rangle+\beta \rho^{P}\left\langle\mathbf{u}^{P}\right\rangle\right]+\boldsymbol{\nabla} \cdot\left[(1-\beta) \rho^{F}\left\langle\mathbf{u}^{F} \mathbf{u}^{F}\right\rangle\right. \\
\left.+\beta \rho^{P}\left\langle\mathbf{u}^{P} \mathbf{u}^{P}\right\rangle\right]=\boldsymbol{\nabla} \cdot\left[(1-\beta)\left\langle\boldsymbol{\sigma}^{F}\right\rangle+\beta\left\langle\boldsymbol{\sigma}^{P}\right\rangle\right]+\rho_{m} \mathbf{g},
\end{aligned}
$$

in which we have assumed the particles to be homogeneous. In this expression, $\mathbf{u}^{P}$ is the local velocity of the particle material and $\boldsymbol{\nabla} \cdot \boldsymbol{\sigma}^{P}$ is the internal force contributing to its time variation. If $\mathbf{u}^{P}$ were replaced by $\mathbf{w}$ so as to make the other definition appear, evidently the right-hand side would have to be modified.

On the basis of the result (22), the average angular momentum balance statement for the mixture may be written as

$$
\begin{aligned}
\frac{\partial}{\partial t}\left\{\mathbf{X} \times\left[(1-\beta) \rho^{F}\left\langle\mathbf{u}^{F}\right\rangle+\beta \rho^{P}\left\langle\mathbf{u}^{P}\right\rangle\right]\right\} \\
+\boldsymbol{\nabla} \cdot\left\{\mathbf{X} \times\left[(1-\beta) \rho^{F}\left\langle\mathbf{u}^{F} \mathbf{u}^{F}\right\rangle+\beta \rho^{P}\left\langle\mathbf{u}^{P} \mathbf{u}^{P}\right\rangle\right]\right\} \\
=\boldsymbol{\nabla} \cdot\left\{\mathbf{X} \times\left[(1-\beta)\left\langle\boldsymbol{\sigma}^{F}\right\rangle+\beta\left\langle\boldsymbol{\sigma}^{P}\right\rangle\right]+\mathrm{C}\right\} \\
\\
\quad+\rho_{m} \mathbf{X} \times \mathbf{g}+n \mathbf{L},
\end{aligned}
$$

where, for simplicity, the external couples acting on the particles have been assumed to be equal and homogeneously distributed over the particle volume. A simple calculation gives 


$$
\begin{aligned}
\mathbf{X} \times\left\{\frac{\partial}{\partial t}\left[(1-\beta) \rho^{F}\left\langle\mathbf{u}^{F}\right\rangle+\beta \rho^{P}\left\langle\mathbf{u}^{P}\right\rangle\right]\right. \\
\left.+\boldsymbol{\nabla} \cdot\left[(1-\beta) \rho^{F}\left\langle\mathbf{u}^{F} \mathbf{u}^{F}\right\rangle+\beta \rho^{P}\left\langle\mathbf{u}^{P} \mathbf{u}^{P}\right\rangle\right]\right\} \\
=\mathbf{X} \times \boldsymbol{\nabla} \cdot\left[(1-\beta)\left\langle\boldsymbol{\sigma}^{F}\right\rangle+\beta\left\langle\boldsymbol{\sigma}^{P}\right\rangle+\rho_{m} \mathbf{g}\right] \\
+\boldsymbol{\nabla} \cdot \mathrm{C}-\beta \boldsymbol{\epsilon} \cdot\left\langle\boldsymbol{\sigma}^{P}\right\rangle+n \mathbf{L} .
\end{aligned}
$$

The terms multiplied by $\mathbf{X}$ cancel by virtue of the momentum equations (B3) and (23) results.

In the previous argument we have assumed that the mass distribution inside the particles is uniform. If this is not the case, the term $\rho_{m} \mathbf{X} \times \mathbf{g}$ in Eq. (B4) becomes more complicated behaving similarly to a nonzero external couple as shown by Almog and Brenner. ${ }^{9}$

\section{APPENDIX C: WEAKLY NONUNIFORM SYSTEM}

Here we extend the analysis of Sec. III to a nonuniform system and we show how the results of Secs. IV and VII can be recovered in this way. We write $\boldsymbol{\sigma}^{P}=\boldsymbol{\sigma}^{P}(x, y, z \mid X, Y, Z)$ with the understanding that the particle center is located at $X, Y, Z$ while $(x, y, z)$ are coordinates relative to this point. Let $z$ be the position of $S_{\text {cut }}$ and $Z_{0}$ the $Z$-coordinate of the surface element $\Delta S$, so that $Z=Z_{0}+z$ (Fig. 3). The integral over $S_{\text {cut }}$ only concerns the variables $x$ and $y$. Let us consider the value of $\boldsymbol{\sigma}^{P}$ at $(x, y, z)$ averaged over all the particles the center of which is at the same level $Z$. The key remark is that this quantity (which we still denote by $\boldsymbol{\sigma}^{P}$ to avoid encumbering the notation) depends strongly on the distance of the integration surface $S_{\text {cut }}$ from the particle center (i.e., the variable $z$ ), while the dependence on $Z$, the position of the particle center itself, occurs over a much slower spatial scale if the nonuniformity of the system is small. Thus we write

$$
\boldsymbol{\sigma}^{P}(x, y, z \mid X, Y, Z)=\boldsymbol{\sigma}^{P}\left(x, y, z \mid X, Y, Z_{0}\right)+\left.z \frac{\partial \boldsymbol{\sigma}^{P}}{\partial Z}\right|_{Z_{0}}+\ldots
$$

The particle number density in Eq. (7) can be expanded in a similar way. We substitute this expansion into Eq. (7) and proceed as shown before in connecting the two sides of Eq. (9) to find, after an obvious transformation to a frameinvariant notation,

$$
\begin{gathered}
\left\langle\sum_{j \in \Delta V_{m}} \int_{S_{\text {cut }}^{j}}\left(\boldsymbol{\sigma}^{P}\right)_{i j} \mathrm{~d} S_{\text {cut }}\right\rangle n(\mathbf{X})\left\langle\int_{v} \boldsymbol{\sigma}_{i j}^{P} \mathrm{~d} v\right\rangle \\
-\frac{\partial}{\partial X_{k}}\left[n(\mathbf{X})\left\langle\int_{v} r_{k} \boldsymbol{\sigma}_{i j}^{P} \mathrm{~d} v\right\rangle\right] .
\end{gathered}
$$

We now decompose the last integral into a symmetric and an antisymmetric part. The latter contributes nothing to the mixture momentum equation which involves the divergence of the stress, i.e., the double divergence over the indices $j$ and $k$ of the quantity in brackets, and we disregard it here. By using an identity similar to Eq. (15) and the particle momentum equation, the remaining term becomes

$$
\begin{aligned}
& \frac{1}{2} \int_{v} {\left[r_{k}\left(\boldsymbol{\sigma}^{P}\right)_{i j}+r_{j}\left(\boldsymbol{\sigma}^{P}\right)_{i k}\right] \mathrm{d} v } \\
& \quad=\frac{1}{2} \oint r_{k} r_{j}\left(\boldsymbol{\sigma}^{F} \cdot \mathbf{n}\right)_{i} \mathrm{~d} S-\frac{1}{2} \int_{v} \rho^{P} r_{k} r_{j}\left(\mathbf{a}^{P}-\mathbf{g}\right) \mathrm{d} v,
\end{aligned}
$$

where, as before, the integration in the first term in the righthand side is over the particle surface. This term can be manipulated to recover the antisymmetric stress and a correction to the symmetric stress as well. The first step is to decompose it according to the representation theory of the rotation group, ${ }^{66}$ as shown in Refs. 4 and 5, to find an expression that can be recast as

$$
\begin{aligned}
T_{i j k}= & \hat{T}_{i j k}+\frac{1}{3} \epsilon_{i j n}\left(\epsilon_{n \ell m} T_{\ell m k}+\epsilon_{k \ell m} T_{\ell m n}\right) \\
& -\frac{1}{5} \epsilon_{i j n} \epsilon_{n k \ell}\left(T_{\ell}-T_{p p \ell}\right)+\frac{1}{5} \delta_{j k} T_{i}+\frac{2}{5} \delta_{i j} T_{p p k}+\Delta_{i j k},
\end{aligned}
$$

where the tensors $T_{i j k}$ and $T_{i}$ are defined in Eq. (29), $\hat{T}_{i j k}$ is the completely symmetric traceless part of $T_{i j k}$, and $\Delta_{i j k}$ is defined in Eq. (28). The first term is a contribution to the symmetric stress arising from spatial nonuniformity, as mentioned in Sec. VII. The second group of terms, which vanish in potential flow, is a contribution to the antisymmetric stress due to a similar effect. Both terms vanish in Stokes flow as shown in Ref. 5. The third term gives rise to the vector $\mathbf{V}$ defined in Eq. (27). The next to the last term is an isotropic contribution which can be considered as a part of the mixture pressure $p_{m}$.

By retaining additional terms in the Taylor series expansion (C1), higher-order tensors $T_{i j k l}$, etc., appear. A corresponding analysis in an ensemble-average framework can be found in Ref. 4. These additional terms, however, are found to have the form of powers of the particle radius multiplied by gradients of averaged quantities as in Eq. (69) and, therefore, vanish in the continuum limit in which the macroscopic length scale becomes much larger than $a$. This circumstance suggests that their importance may be limited in most situations.

\section{APPENDIX D: SYMMETRIC STRESS}

It has been shown in earlier work ${ }^{4,67}$ that, in general, the symmetric part of the stress has the form

$$
\langle\boldsymbol{\tau}\rangle^{S}=2 \mu \mathrm{E}_{m}+n\langle\mathrm{~S}\rangle+\boldsymbol{\nabla} \cdot\left[n\left\langle\mathrm{~S}^{(3)}\right\rangle+\boldsymbol{\nabla} \cdot\left(n\left\langle\mathrm{~S}^{(4)}\right\rangle+\ldots\right)\right],
$$

where $S$ is the stresslet and $S^{(3)}, S^{(4)}, \ldots$ are average symmetric multipoles of order higher than two. While the contribution of these higher-order terms evidently vanishes in a uniform system, it is not necessarily so in the presence of nonuniformities. A relatively straightforward calculation accurate to $O(\beta)$ suggests that, in the Stokes regime, Eq. (D1) takes the form of Eq. (41). The closure parameters $\mu_{\mathrm{eff}}, \mu_{\Delta}$, and $\mu_{\nabla}$ can be calculated numerically in the same way described in Refs. 5, 68, and 69.

For this purpose, for each value of the volume fraction, we construct ensembles (each one consisting of between 256 and 2048 different configurations) consisting of $N$ particles 
(with $N$ ranging between 10 and 160) randomly arranged in a periodic cubic cell of side $L$. Each configuration is generated by subjecting the particles to a large number of random displacements. Examples of the pair distribution function, nearest-neighbor distribution, and static structure factor of the ensembles that we use are given in the cited references and show a satisfactory degree of randomness and statistical uniformity. By considering, for each volume fraction, bigger and bigger cells, we extrapolate the results to infinite system size $L / a \rightarrow \infty$.

We simulate two different physical situations in which the particles are immersed in a viscous fluid with vanishing inertial effects. For the first one, we use a uniform ensemble. Each particle in each configuration is subjected to a positiondependent force $\mathbf{f}^{\alpha}=6 \pi \mu a \mathbf{w}_{0} \sin \mathbf{k} \cdot \mathbf{y}^{\alpha}$ where $\mathbf{w}_{0}$ is a constant vector parallel to one of the three sides of the fundamental cell; $\mathbf{k}$ is taken in turn parallel to each side of the cell, and $|\mathbf{k}|=2 \pi / L$. With the results of this simulation we are able to calculate $\mu_{\text {eff }}$ and $\mu_{\Delta}$, but not $\mu_{\nabla}$ given that $\mathrm{E}_{\nabla}=0$ for a uniform ensemble. For the second simulation the particles are subjected to a constant force, but we use a nonuniform ensemble constructed, as described in Refs. 68 and 70, so that $\boldsymbol{\nabla} \beta \neq 0$ and $\mathrm{E}_{\nabla} \neq 0$ as well. These simulations enable us to obtain $\mu_{\nabla}$ together with a second estimate of $\mu_{\mathrm{eff}}$.

The many-body problem is solved by a multipole method described in Ref. 71 and adapted as described in Refs. 68 and 70. The multipole expansion included terms up to the fifth order for $\beta<40 \%$; for $\beta=40 \%$ and $45 \%$ one more order was included. Further details on this work will be presented in a future paper.

${ }^{1} \mathrm{C}$. Truesdell, "The mechanical foundations of elasticity and fluid dynamics," J. Rational Mech. Anal. 1, 125 (1952).

${ }^{2}$ J. Serrin, in Handbuch der Physik, edited by C. Truesdell (Springer, Berlin, 1959), Vol. VIII/1, p. 133.

${ }^{3}$ S. Aris, Vectors, Tensors, and the Basic Equations of Fluid Mechanics (Prentice-Hall/Dover, Englewoof Cliffs, NJ, 1962).

${ }^{4}$ A. Prosperetti, "The average stress in incompressible disperse flow," Int. J. Multiphase Flow 30, 1011 (2004).

${ }^{5}$ A. Prosperetti, Q. Zhang, and K. Ichiki, "The stress system in a suspension of heavy particles: Antisymmetric contribution," J. Fluid Mech. 554, 125 (2006).

${ }^{6}$ L. Landau and E. Lifshitz, Fluid Mechanics, 2nd ed. (Pergamon, New York, 1987).

${ }^{7}$ G. Batchelor, "The stress system in a suspension of force-free particles," J. Fluid Mech. 41, 545 (1970).

${ }^{8}$ H. Brenner, "Rheology of two-phase systems," Annu. Rev. Fluid Mech. 2, 137 (1970).

${ }^{9}$ Y. Almog and H. Brenner, "Ensemble-average versus suspension-scale Cauchy continuum-mechanical definitions of stress in polarized suspensions: Global homogenization of a dilute suspension of dipolar spherical particles," Phys. Fluids 11, 268 (1999).

${ }^{10} \mathrm{~B}$. U. Felderhof, "The effect of Brownian motion on the transport properties of a suspension of spherical particles," Physica A 118, 69 (1983).

${ }^{11}$ B. Cichocki, B. U. Felderhof, and R. Schmitz, "The effective viscosity of suspensions and emulsions of spherical particles," Physica A 154, 233 (1989).

${ }^{12} \mathrm{C}$. Beenakker, "The effective viscosity of a concentrated suspension of spheres (and its relation to diffusion)," Physica A 128, 48 (1984).

${ }^{13}$ D. Bedeaux, "The effective viscosity for a suspension of spheres," J. Colloid Interface Sci. 118, 80 (1987).

${ }^{14}$ R. Herczynski and I. Pienkowska, "Toward a statistical theory of suspension,” Annu. Rev. Fluid Mech. 12, 237 (1980).

${ }^{15}$ J. K. G. Dhont and W. J. Briels, "Stresses in inhomogeneous suspensions," J. Chem. Phys. 117, 3992 (2002).
${ }^{16}$ L. G. Leal, "On the effect of particle couples on the motion of a dilute suspension of spheroids," J. Fluid Mech. 46, 395 (1971).

${ }^{17} \mathrm{H}$. Brenner, "Suspension rheology in the presence of rotary Brownian motion and external couples: Elongational flow of dilute suspensions," Chem. Eng. Sci. 27, 1069 (1972).

${ }^{18}$ H. Brenner, "Vortex flows," Int. J. Eng. Sci. 22, 645 (1984).

${ }^{19}$ S. Feng, A. L. Graham, J. R. Abbott, and H. Brenner, "Antisymmetric stresses in suspensions: Vortex viscosity and energy dissipation,” J. Fluid Mech. 563, 97 (2006).

${ }^{20} \mathrm{G}$. Wallis, "The average Bernoulli equation and macroscopic equations of motion for the potential flow of a two-phase dispersion," Int. J. Multiphase Flow 17, 683 (1991).

${ }^{21}$ A. Sangani and A. Didwania, "Dispersed-phase stress tensor in flows of bubbly liquids at large Reynolds numbers," J. Fluid Mech. 248, 27 (1993).

${ }^{22}$ A. Biesheuvel and L. van Wijngaarden, "Two-phase flow equations for a dilute dispersion of gas bubbles in liquid," J. Fluid Mech. 148, 301 (1984).

${ }^{23}$ D. Drew and R. J. Lahey, in Particulate Two-Phase Flow, edited by M. C. Roco (Butterworth, Boston, 1993), pp. 509-566.

${ }^{24} \mathrm{D}$. Zhang and A. Prosperetti, "Averaged equations for inviscid disperse two-phase flow," J. Fluid Mech. 267, 185 (1994).

${ }^{25}$ D. Zhang and A. Prosperetti, "Ensemble phase-averaged equations for bubbly flows," Phys. Fluids 6, 2956 (1994).

${ }^{26} \mathrm{H}$. Bulthuis, A. Prosperetti, and A. Sangani, “Particle stress' in disperse two-phase potential flow," J. Fluid Mech. 294, 1 (1995).

${ }^{27}$ S. Kang, A. Sangani, H. Tsao, and D. Koch, "Rheology of dense bubble suspensions," Phys. Fluids 9, 1540 (1997).

${ }^{28} \mathrm{R}$. O'Brien, "A method for the calculation of the effective transport properties of suspensions of interacting particles," J. Fluid Mech. 91, 17 (1979).

${ }^{29} \mathrm{~S}$. Whitaker, "Advances in theory of fluid motion in porous media," Ind. Eng. Chem. 61, 14 (1969).

${ }^{30}$ M. Ishii, Thermo-Fluid Dynamic Theory of Two-Phase Flow (Eyrolles, Paris, 1975).

${ }^{31}$ R. Nigmatulin, "Spatial averaging in the mechanics of heterogeneous and dispersed systems," Int. J. Multiphase Flow 5, 353 (1979).

${ }^{32}$ If $\sigma^{P}$ is not symmetric, the error incurred is of the order of $(\beta / v-n) \mathbf{L}$, where $\mathbf{L}$ is the external couple on the particles introduced later.

${ }^{33}$ The fundamental difference between the two phases is that, for the particles, the stress depends on the distance from the particle center, while this notion is meaningless for the fluid. In other words, the finite size of the particles introduces nonlocal effects at the microscale, which are absent for the fluid.

${ }^{34}$ Z. Zhang and A. Prosperetti, "A method for three-dimensional particle flow simulations," J. Comput. Phys. 210, 292 (2005).

${ }^{35}$ J. Bear, Dynamics of Fluids in Porous Media (Academic, New York, 1972).

${ }^{36} \mathrm{~S}$. Whitaker, The Method of Volume Averaging (Kluwer, Dordrecht, 1999).

${ }^{37}$ It is true that Batchelor goes on to show that, in his situation, the average over a surface cutting the particles equals that over a boundary, which does not, but this is a consequence of the momentum theorem and cannot be found from averaging alone.

${ }^{38} \mathrm{G}$. Batchelor, "A new theory of the instability of a uniform fluidized bed," J. Fluid Mech. 193, 75 (1988).

${ }^{39}$ J. Brady, "The rheological behavior of concentrated colloidal dispersions," J. Chem. Phys. 99, 567 (1993).

${ }^{40}$ In writing the first term $\rho_{m} \partial \mathbf{u}_{m} / \partial t$ we assume that the difference between the center of mass velocity and $\mathbf{u}_{m}$ is small.

${ }^{41}$ As shown below, the Reynolds number in this simulation is smaller than 1 , which would permit us to drop the time derivative term. The argument to retain it may be described as follows. The steady momentum equation can be written in shorthand as $L u=0$, where $L$ is a suitable linear operator. A standard way to solve a problem of this type is to use an iterative scheme $u^{n+1}=u^{n}+\delta L u^{n}$, where $\delta$ is a relaxation parameter. Evidently, as $n \rightarrow \infty, u^{n}$ converges to the solution of $L u=0$. If $u^{n}$ is close to $u^{n+1}$, one iteration is sufficient for an acceptable degree of convergence. Such a numerical procedure is equivalent to a time-marching scheme, which is essentially our method. As a check, we have adapted the same numerical method to an equation including the convective term $\mathbf{u}_{m} \cdot \nabla \mathbf{u}_{m}$ finding results with a negligible difference.

${ }^{42} \mathrm{H}$. Brenner and V. Ganesan, "Molecular wall effects: Are conditions at a boundary 'boundary conditions'?” Phys. Rev. E 61, 6879 (2000). 
${ }^{43}$ A. Prosperetti and G. Tryggvason, Computational Methods for Multiphase Flow (Cambridge University Press, Cambridge, 2007).

${ }^{44}$ J. M. Nitsche and G. K. Batchelor, "Break-up of a falling drop containing dispersed particles," J. Fluid Mech. 340, 161 (1997).

${ }^{45}$ M. L. Ekiel-Jezewska, B. Metzger, and E. Guazzelli, "Spherical cloud of point particles falling in a viscous fluid," Phys. Fluids 18, 038104 (2006).

${ }^{46}$ B. Metzger, M. Nicolas, and E. Guazzelli, "Falling clouds of particles in viscous fluids," J. Fluid Mech. 580, 283 (2007).

${ }^{47}$ H. Lamb, Hydrodynamics, 6th ed. (Cambridge University Press, Cambridge, 1932).

${ }^{48} \mathrm{H}$. Brenner, "Rheology of a dilute suspension of dipolar spherical particles in an external field," J. Colloid Interface Sci. 32, 141 (1970).

${ }^{49}$ A. C. Eringen, Nonlocal Continuum Field Theories (Springer, New York, 2002).

${ }^{50}$ J. D. Goddard, in Mathematical Models for Granular Matter, Lecture Notes in Mathematics, edited by G. Capriz, P. Giovine, and P. M. Mariano (Springer, New York, 2008), Vol. 1937, pp. 1-22.

${ }^{51}$ J. P. Bardet and I. Verdoulakis, "The asymmetry of stress in granular media," Int. J. Solids Struct. 38, 353 (2001).

${ }^{52}$ D. Bigoni and W. J. Drugan, "Analytical derivation of Cosserat moduli via homogenization of heterogeneous elastic materials," J. Appl. Mech. 74, 741 (2007).

${ }^{53}$ K. S. Ananda, J. Patra, and P. R. Nott, "Experimental evidence of the kinematic Cosserat effect in dense granular flows," Phys. Fluids 21, 051703 (2009).

${ }^{54}$ G. K. Batchelor and J. Green, "The determination of the bulk stress in a suspension of spherical particles to order $c^{2}$," J. Fluid Mech. 56, 401 (1972).

${ }^{55}$ F. Gadala-Maria and A. Acrivos, "Shear-induced structure in a concentrated suspension of solid spheres," J. Rheol. 24, 799 (1980).

${ }^{56}$ J. Brady and J. Morris, "Microstructure of strongly sheared suspensions and its impact on rheology and diffusion," J. Fluid Mech. 348, 103 (1997).

${ }^{57}$ J. Morris and B. Katyal, "Microstructure from simulated Brownian suspension flows at large shear rate," Phys. Fluids 14, 1920 (2002).

${ }^{58}$ W. B. Russel and A. P. Gast, "Nonequilibrium statistical mechanics of concentrated colloidal dispersions: Hard spheres in weak flows," J. Chem. Phys. 84, 1815 (1986).
${ }^{59}$ J. Morris and F. Boulay, "Curvilinear flow of noncolloidal suspensions: The role of normal stresses," J. Rheol. 43, 1213 (1999).

${ }^{60}$ I. Zarraga and D. Leighton, "Normal stress and diffusion in a dilute suspension of hard spheres undergoing simple shear," Phys. Fluids 13, 565 (2001).

${ }^{61}$ A. Sierou and J. F. Brady, "Rheology and microstructure in concentrated noncolloidal suspensions," J. Rheol. 46, 1031 (2002).

${ }^{62}$ J. H. Irving and J. G. Kirkwood, "The statistical mechanical theory of transport processes. IV. The equations of hydrodynamics," J. Chem. Phys. 18, 817 (1950).

${ }^{63} \mathrm{G}$. K. Batchelor, "The effect of Brownian motion on the bulk stress in a suspension of spherical particles," J. Fluid Mech. 83, 97 (1977).

${ }^{64}$ P. R. Nott and J. F. Brady, "Pressure-driven flow of suspensions: Simulation and theory," J. Fluid Mech. 275, 157 (1994).

${ }^{65}$ If, accounting for collisions, we write $\boldsymbol{\sigma}^{P}=\boldsymbol{\sigma}+\boldsymbol{\sigma}_{\text {coll }}$, and model the second term as a localized impulse, the collisional contribution to the mixture stress becomes $\left(\left\langle\boldsymbol{\sigma}^{P}\right\rangle\right)=\left\langle\mathbf{r} \mathbf{F}_{\text {coll }}\right\rangle$ in which $\mathbf{F}_{\text {coll }}$ is the force due to the collision. In this way we recover the standard expression of the collisional stress in a granular material [see, e.g., C. S. Campbell, "Granular material flows-An overview," Powder Technol. 162, 208 (2006)].

${ }^{66} \mathrm{~T}$. Damour and B. Iyer, "Multipole analysis for electromagnetism and linearized gravity with irreducible Cartesian tensors," Phys. Rev. D 43, 3259 (1991).

${ }^{67} \mathrm{M}$. Tanksley and A. Prosperetti, "Average pressure and velocity fields in non-uniform suspensions of spheres in Stokes flow," J. Eng. Math. 41, 275 (2001).

${ }^{68} \mathrm{~K}$. Ichiki and A. Prosperetti, "Faxén-like relations for a non-uniform suspension," Phys. Fluids 16, 2483 (2004).

${ }^{69} \mathrm{Q}$. Zhang, K. Ichiki, and A. Prosperetti, "On the computation of ensemble averages for spatially non-uniform particle systems," J. Comput. Phys. 212, 247 (2006)

${ }^{70}$ M. Marchioro, M. Tanksley, and A. Prosperetti, "Flow of spatially nonuniform suspensions. Part I: Phenomenology," Int. J. Multiphase Flow 26, $783(2000)$

${ }^{71}$ G. Mo and A. Sangani, "A method for computing Stokes flow interactions among spherical objects and its application to suspensions of drops and porous particles," Phys. Fluids 6, 1637 (1994). 